\title{
Catch the star! Spatial information activates the manual motor system
}

\author{
A. Miklashevsky ${ }^{*}$ \\ ${ }^{1}$ Potsdam Embodied Cognition Group, University of Potsdam, Germany
}

*Correspondence:

Alex Miklashevsky

armanster31@gmail.com 


\begin{abstract}
Previous research demonstrated a close bidirectional relationship between spatial attention and the manual motor system. However, it is unclear whether an explicit hand movement is necessary for this relationship to appear. A novel method with high temporal resolution - bimanual grip force registration - sheds light on this issue. Participants held two grip force sensors while being presented with lateralized stimuli (exogenous attentional shifts, Experiment 1), left- or right-pointing central arrows (endogenous attentional shifts, Experiment 2), or the words "left" or "right" (endogenous attentional shifts, Experiment 3). There was an early interaction between the presentation side or arrow direction and grip force: lateralized objects and central arrows led to an increase of the ipsilateral force and a decrease of the contralateral force. Surprisingly, words led to the opposite pattern: increased force in the contralateral hand and decreased force in the ipsilateral hand. The effect was stronger and appeared earlier for lateralized objects (60 ms after stimulus presentation) than for arrows (100 ms) or words $(250 \mathrm{~ms})$. Thus, processing visuospatial information automatically activates the manual motor system, but the timing and direction of this effect vary depending on the type of stimulus.
\end{abstract}

Keywords: spatial attention, spatial processing, endogenous attention, exogenous attention, motor system, grip force 


\section{Catch the star! Spatial information activates the manual motor system}

\section{Introduction}

The manual motor system and orienting of spatial attention are closely related. Eye-hand coordination enables efficient reaching, grasping, and object manipulation. This coordination requires analyzing visual information about the environment surrounding hands and simultaneous control of ongoing hand movements (see for review Rizzo et al., 2017).

Attention can prioritize spatial locations already before the execution of a hand movement. This effect was shown in a series of experiments where participants pointed at different locations on a screen and then reported a briefly presented target symbol (Baldauf et al., 2006; Baldauf \& Deubel, 2008; see also Festman et al., 2013). Notably, the target symbol was presented either in a position later pointed at or in a different position. Participants were significantly more accurate at identifying the target symbol when it was presented in a to-be-pointed-at location. Eye-hand coordination is a complex bidirectional process and not merely serial delivery of motor commands to the execution level after complete analysis of visual information. Multiple target coordinates are averaged and influence the ongoing movement, constantly updating the motor program (Chapman et al., 2010; Gallivan \& Chapman, 2014; see Hommel et al., 2019, for a theoretical account).

In line with this research is the so-called near-hand effect (Abrams et al., 2008): attention is more engaged when objects appear close to hands. Participants more efficiently inhibit distractors presented farther away from hands (Davoli \& Brockmole, 2012) and identify new objects in the near-hand space faster (Reed et al., 2006; see Brockmole et al., 2013, for a review). Hand movements lead to dynamic changes of the near-hand space, which results in faster detection of targets closer to hands at every moment (Adam et al., 2012). Also, the task and type of stimuli matters: having one's hand close to words or arithmetical expressions is disadvantageous for semantic processing of those symbolic stimuli (Davoli et al., 2010, 2020). 
bioRxiv preprint doi: https://doi.org/10.1101/2021.1228.474381; this version posted December 28,2021 . The copyright holder for this preprint (which was not certified by peer review) is the author/funder, who has granted bioRxiv a license to display the preprint in perpetuity. It is made available under aCC-BY 4.0 International license.

Thus, previous research convincingly demonstrated a bidirectional relationship between spatial attention and hand movement across various paradigms. But how automatic is this link between the manual motor system and spatial processing? Is hand movement, whether ongoing or potential, a necessary component for this relationship to appear? In a previous study, my colleagues and I presented participants with large vs. small numerical stimuli (Miklashevsky et al., 2021) while monitoring participants' spontaneous hand motor activity using two grip force sensors (Aravena et al., 2012, 2014; da Silva et al., 2019; Frak et al., 2010; Pérez-Gay Juárez et al., 2019; for review and methodological details, see Nazir et al., 2017). No manual response or explicit hand movement was required. Numerical cognition research shows that small numbers are associated with the left peripersonal space, and large numbers are associated with the right peripersonal space (Spatial Numerical Association of Response Codes, or SNARC effect, see Dehaene et al., 1993). This effect has been demonstrated with button press responses, finger movements (Fischer, 2003), eye movements (Myachykov et al., 2016), foot responses (Schwarz \& Müller, 2006), and even full-body movements (see for reviews Fischer \& Shaki, 2014; Toomarian \& Hubbard, 2018; see for meta-analysis Wood et al., 2008). Despite this overwhelming evidence for the presence of spatial-numerical associations, we found no SNARC effect in grip force (Miklashevsky et al., 2021), either because no attentional shifts appeared without explicit motor responses to numeric stimuli (cf. Shaki \& Fischer, 2018) or because the manual motor system is not activated automatically by spatial information. The latter hypothesis was tested explicitly in the present study.

The goal of the present study was to investigate the direct effects of attentional shifts on the manual motor system in the absence of any motor action. A novel method, bimanual grip force recording, allows monitoring motor activity in hands with millisecond resolution while participants process visual stimuli. Grip force sensors measure spontaneous motor activity during action observation (Blampain et al., 2018) and semantic processing of motor-related language (Aravena et al., 2012, 2014; da Silva et al., 2019; Frak et al., 2010; Pérez-Gay Juárez et al., 2019). Both hands exhibit comparable activity in response to motor-related linguistic stimuli (da Silva et al., 2018). 
In the present study, I recorded grip force bimanually while manipulating participants' visual attention. Specifically, exogenous and endogenous attentional shifts (see Klein, 2004; Posner, 1980) were induced using different types of stimuli. Lateralized stimuli cause exogenous attentional shifts by summoning attention as they are physically present at a particular location. In the present study, exogenous attentional shifts were induced by presenting participants with left- or right-localized stars (Experiment 1). In contrast, centrally presented symbolic stimuli direct attention by their meaning and thus lead to endogenous attentional shifts. In the present study, endogenous attentional shifts were induced by presenting participants with left- or right-pointing arrows (Experiment 2) or words "left" and "right" (Experiment 3). Additionally, bimanual grip force recording has a high temporal resolution $(1000 \mathrm{~Hz})$, making it possible to reveal the precise timing of manual motor activity accompanying attentional shifts.

\section{GENERAL METHOD}

Forty-two psychology and linguistics students (14 males, 28 females) of the University of Potsdam participated in the study for course credit. An entire testing session consisted of four experiments: lateralized star presentation (Experiment 1), lateralized sound presentation (not reported here), central arrow presentation (Experiment 2), and word presentation (Experiment 3). All experiments were conducted in a pseudorandom order to exclude possible systematic sequence or fatigue effects. The exact order of experiments is specified for every participant in supplementary materials (see data availability statement). Participants were allowed to take breaks between experiments, walk and drink water. The whole testing session lasted between 60 and 90 minutes.

After the experiments, participants completed questionnaires including demographic data (gender, age, native language, and foreign languages they speak) and physiological data (seeing problems, hearing problems, motor diseases, and whether they take medications that can influence motor control). Additionally, participants filled in the Edinburg Handedness Inventory (EHI, Oldfield, 1971), where original instructions were replaced with a more intuitive Likert scale as suggested elsewhere 
(Robinson, 2013). Resulting EHI scores range from +100 (exclusively right-handed) through 0 (ambidextrous) to -100 (exclusively left-handed). All participants signed an informed consent form before the study. The local Ethics Committee approved the study (study number 15/2019).

One participant was excluded due to a self-reported motor problem (light essential tremor) clearly reflected in his force data. Another participant reported left leg paralysis, but her data were not qualitatively different from the rest of the sample and thus remained in the final dataset. Only German native speakers participated in the word presentation study (Experiment 3). All but one participant reported having normal or corrected-to-normal vision.

\section{Equipment and data acquisition}

The method followed closely the one recommended by Nazir et al. (2017) for single-sensor recording.

Both sensors were stand-alone load cells manufactured by ATI Industrial Automation, USA (www.atiia.com/Products/ft/sensors.aspx). They resembled large metal coins with $40 \mathrm{~mm}$ diameter and $14 \mathrm{~mm}$ height, and each weighed $57 \mathrm{~g}$. Each sensor was covered from both contact sides with a $3 \mathrm{~mm}$ plastic cover of the same diameter as the sensor itself $(40 \mathrm{~mm})$, resulting in a total thickness of $20 \mathrm{~mm}$ and a total weight of $65 \mathrm{~g}$ per sensor (see Figure 1). These sensors record force dynamics with millisecond resolution along three orthogonal axes, but only $\mathrm{Fz}$ force along the vertical axis through the sensors was analyzed and reported here. Two PCs were used: one for running the experiment under OpenSesame software (Mathôt et al., 2012) and another for force data acquisition under Expyriment software (Krause \& Lindemann, 2014). The first PC sent a trigger at the beginning of each trial to later identify a corresponding time point in the force data file. Monitor Philips Brilliance LCD 220P4LPY $22 "$ with a screen resolution $1680 \times 1050$ was used for stimuli presentation. 


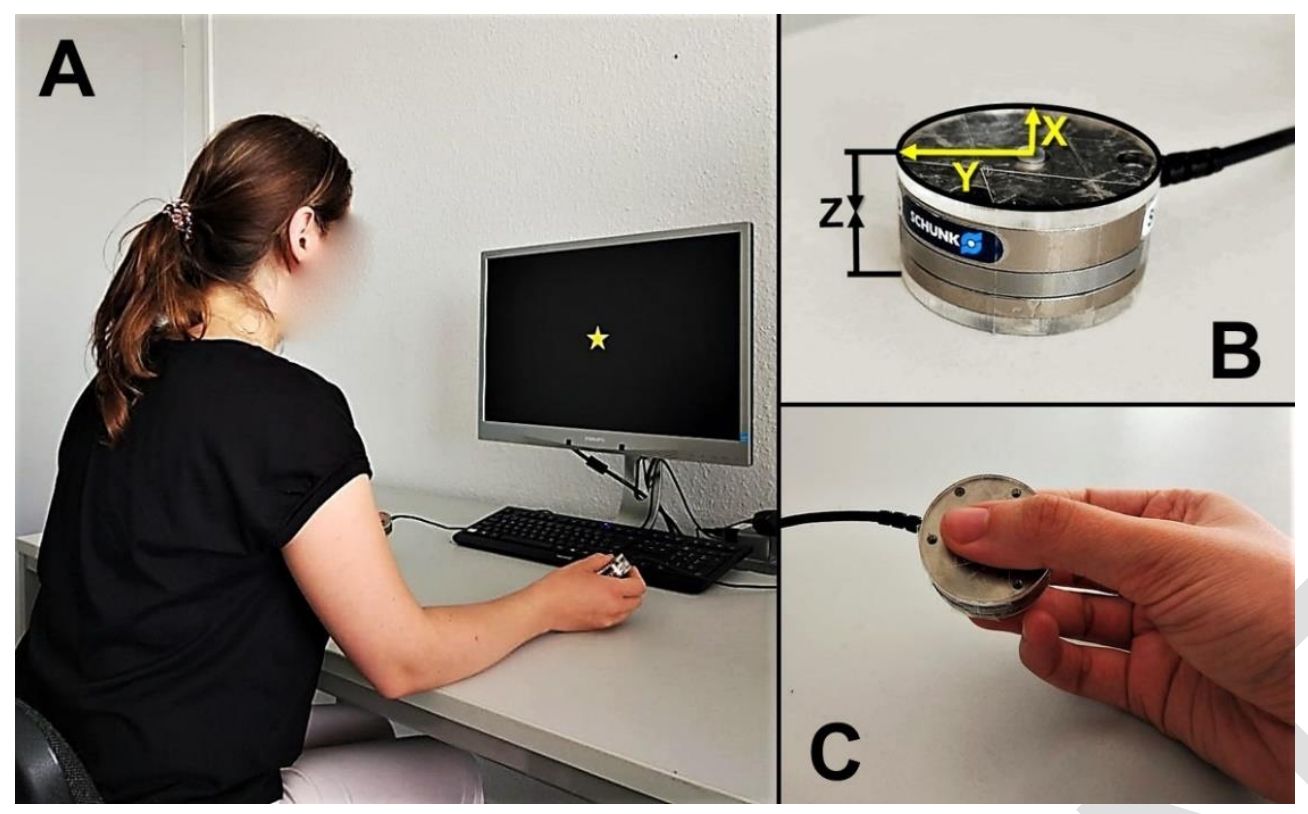

Fig. 1. Experimental setup and equipment. Panel A. Bimanual force recording setup. Panel B. Grip force sensor (X - longitudinal, Y - radial, $\mathrm{Z}$ - compression forces). C. The way participants held the sensors (around $45^{\circ}$ relative to the table surface, not strictly controlled). Adapted image from Miklashevsky et al. (2021, Fig. 2).

Participants sat at a desk and held one sensor in each hand at an angle of around $45^{\circ}$ relative to the table surface with the thumb on one side and the index and middle fingers on the other side.

Participants' elbows rested on the table while their hands held the sensors, thus preventing sensor slippage (see Fig. 1). The distance between sensors varied from 30 to $50 \mathrm{~cm}$ and was not strictly controlled, but both were equidistant from each participant's mid-sagittal plane. The distance from participants' eyes was around $60 \mathrm{~cm}$.

Before data collection, participants practiced applying a holding force in a range between $1.5 \mathrm{~N}$ and 3 $\mathrm{N}$ with each hand. The sensors were represented on the screen as two circles that changed their color from green ("too weak") to red ("too strong") with the pre-defined force range indicated by the grey color. As soon as participants managed to turn both circles into grey, they received an instruction to keep the force at this level during the whole testing session. Data collection started automatically after 
participants held the sensor with the required force for three seconds without crossing these thresholds.

This calibration procedure was repeated after each break and at the beginning of each experiment.

\section{Experiment 1: Star presentation}

Participants were presented with either central or lateralized (left vs. right) visual stimuli in this experiment. This ensured exogenous control over attention (Posner, 1980), while bimanual force recording allowed investigating the dynamic involvement of the motor system in the processing of spatial information.

\section{Participants}

Forty-one psychology and linguistics students (13 males, 28 females; mean age $=24$ years $)$

participated in the experiment. Their mean EHI score was 66 (80\% had EHI score > 50; $10 \%$ had EHI score between +50 and $-50 ; 10 \%$ had EHI score < -50). All but one participant reported normal or corrected-to-normal vision. No participant took medications affecting motor control.

\section{Stimuli and design}

Red and yellow stars were used as stimuli in catch (go) and critical (no-go) trials accordingly. The background was kept black. Stimuli can be found in the supplementary data (see data availability statement). Stars appeared in the middle of the screen, on the left or the right side. Grip force was recorded bimanually. This results in a 2 (Hand: left / right) X 3 (Position of the star: left / central / right) within-participant design.

\section{Task and procedure}

After the calibration procedure described above, the experiment started. Each trial consisted of a fixation dot (200 ms), followed by a stimulus (until response, but no longer than $2000 \mathrm{~ms}$ ). The stimuli were stars around $4 \mathrm{~cm}$ in diameter (3.82 degrees of visual angle calculated by using the formula $57.3^{*} \mathrm{w} / \mathrm{d} ; \mathrm{w}-$ width of the object; $\mathrm{d}$ - distance to the object), which appeared with equal probability $(33 \%)$ in one of three positions: at the center of the screen, or $19.5 \mathrm{~cm}$ left or right from the center 
bioRxiv preprint doi: https://doi.org/10.1101/2021.1228.474381; this version posted December 28, 2021. The copyright holder for this preprint (which was not certified by peer review) is the author/funder, who has granted bioRxiv a license to display the preprint in perpetuity. It is made available under aCC-BY 4.0 International license.

(18.62 degrees of visual angle). $75 \%$ of the stars were yellow, and red stars appeared in $25 \%$ of all trials. The task was to say "yes" when a red star appeared, regardless of stimulus location. Participants were asked not to rotate their heads when stars appeared laterally, but eye movements were allowed.

Additionally, participants were instructed not to cross their legs during the experiment. Critical trials for analysis were no-go trials (yellow stars). This means that overt motor or verbal responses do not contaminate grip force recordings. Such responses typically generate large artifacts in these recordings (e.g., see panel A at Figures 2, 4, and 6). The experiment consisted of 360 trials with a break in the middle and lasted around 15 minutes. It was preceded by a short practice (12 trials).

\section{Data preprocessing and analysis}

The preprocessing of grip force data closely followed the recommendations of Nazir et al. (2017, Experiment 2). Data were filtered at $15 \mathrm{~Hz}$ before analysis with a fourth-order, zero-phase, lowpass Butterworth filter. Single epochs were extracted from the vertical Fz signal, starting $200 \mathrm{~ms}$ before and ending $1000 \mathrm{~ms}$ after stimulus onset. The global drift in force across the experiment was corrected by subtracting the average force from $20 \mathrm{~ms}$ intervals before stimulus onset from each epoch. As a result, grip force always crosses the zero point at the start of each trial, and negative force values reflect a vertical grip force less than that at the moment of stimulus presentation, not the absence of force. Maximum and minimum thresholds were applied $( \pm 500 \mathrm{mN})$ to remove movement artifacts and identify participants with unacceptably large force variability. The proportion of trials where force exceeded one of the thresholds varied across participants from $0 \%$ to $19 \%$ (mean $=2 \%$ ). Those trials were discarded, but no participant was excluded due to this criterion. Accuracy varied from $98 \%$ to $100 \%($ mean $=100 \%)$; error trials were excluded from further analysis.

Overall grip force patterns are presented in Figure 2 to give readers an overview of this relatively unfamiliar data type. The blue line in Figure 2A represents averaged force of both hands for all accepted no-go trials. As suggested before (Miklashevsky et al., 2021), these changes will be referred to as $\mathrm{H}$ (high force, peaks) and $\mathrm{L}$ (low force, dips), with a number representing a time point. 
For example, H130 means a peak with its highest point at around $130 \mathrm{~ms}$, whereas L450 denotes a dip with its lowest point at $450 \mathrm{~ms}$ after stimulus onset. As one can see, independently of a particular condition, grip force follows the following pattern: there are three peaks (H130, H330, and H600) with two dips between them (L230 and L450). The second peak (H330) is the tallest. Figure 2B represents averaged grip force changes in go (dotted red line) and no-go (solid blue line) trials. These two lines start diverging at $250 \mathrm{~ms}$ after stimulus onset with force in go trials reaching its highest point (almost $50 \mathrm{mN}$ ) at around $750 \mathrm{~ms}$ and remaining at this level till the end of the epoch $(1000 \mathrm{~ms})$. Figure $2 \mathrm{C}$ represents force averaged by condition (Hand and Position): red lines represent right-hand forces, blue lines represent left-hand forces; dotted lines represent the star-left condition, solid lines the star-in-thecenter, dashed lines the star-right condition. 

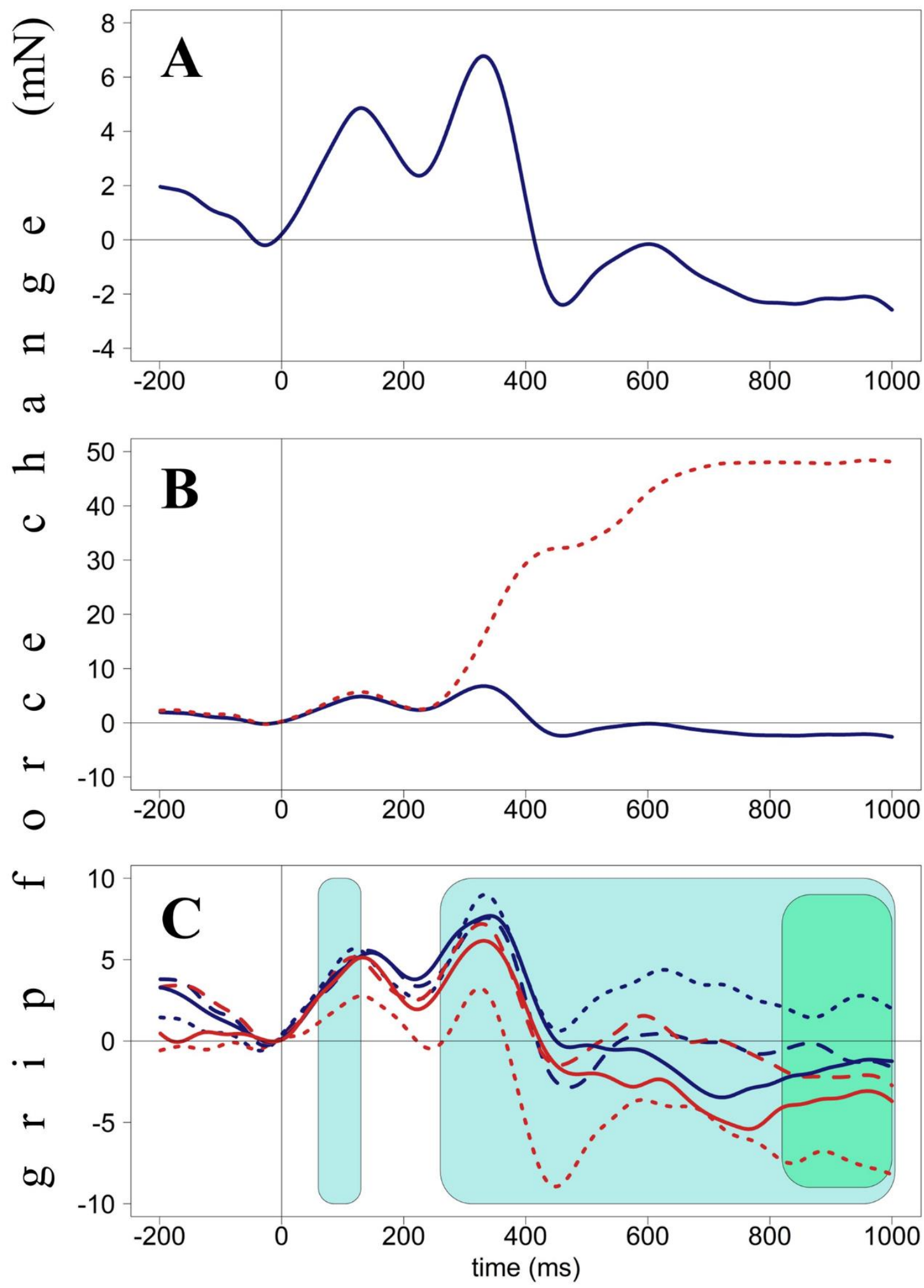

Fig. 2. Grip force changes (in milli-Newton) plotted against time from stimulus onset (in

milliseconds), Experiment 1. Panel A. Averaged force profiles across all accepted no-go trials of all participants. Panel B. Force profiles in go (dotted red line) and no-go (solid blue line) trials. These 
forces diverge around $250 \mathrm{~ms}$ after stimulus presentation. Panel C. Force profiles averaged by condition (Hand X Position). Light-blue areas (60-130 ms and 260-1000 ms) indicate interactions between Hand and Position, green area (820-1000 ms) indicates the main effect of Hand. Red lines represent right-hand forces, blue lines - left-hand forces. Dotted lines represent the star-left condition, solid lines - star-in-the-center condition, dashed lines - star-right condition.

The method used by Miklashevsky et al. (2021) was applied to identify time windows of interest: forces were aggregated by Hand (left / right) and Position (right/central/left) within participants. With Hand and Position as within-variables, these data were submitted to a cluster permutation analysis ( $\mathrm{R}$ Core Team, 2020; package "permuco", see Frossard \& Renaud, 2018). Five thousand permutations were performed, and TFCE (Threshold-Free Cluster Enhancement) correction for multiple comparisons was used. The analysis revealed four time windows with significant or close to significance effects: main effect of Hand (820-1000 ms after stimulus onset), main effect of Position (680-760 ms), and two time windows with interactions between Hand and Position (60-130 ms and 260-1000 ms after stimulus onset).

820-1000 ms, Model 1.1. The data (averaged by Hand and Position for each participant) were then submitted to a linear mixed model analysis using the lme4 package (Bates et al., 2015) in R. The categorical predictor Hand was sum-coded (left/right, sum-coded contrast -0.5 and 0.5, see Barr et al., 2013). Position was re-coded in a continuous manner (left: -1; center: 0; right: 1). Interaction between Hand and Position was included. Participants were included as random factors. I performed a backward elimination using the drop1 function to identify the best-fit model; effects and interactions that did not improve model fit $(\mathrm{p}>.1)$ were successively eliminated. Only significant effects are reported unless the effect of interest in a given analysis was non-significant - in such cases, it is also reported. 
The main effect of Hand was significant: grip force in the left hand was more prominent than in the right hand $(\mathrm{b}=-4.261, \mathrm{p}=.001)$. The interaction between Hand and Position was also significant $(\mathrm{b}=$ $4.071, \mathrm{p}=.009)$. Still, since this time window is a part of a larger one $(260-1000 \mathrm{~ms})$ suggested by the cluster permutation analysis for this interaction, it was not examined further. Marginal r-squared (variance explained by fixed effects, see Nakagawa \& Schielzeth, 2013) was .041, and conditional rsquared (variance explained by the whole model) was .451. See Table 1 for further details; see also Figure 2C.

Table 1. Model 1.1. Main effect of Hand on grip force in the time window 820-1000 ms (Experiment 1).

\begin{tabular}{lcccc}
\hline Random effects: & Name & Variance & SD & \\
\hline Participants & Intercept & 74.890 & 8.654 & \\
Residual & & 100.180 & 10.009 & \\
\hline Fixed effects: & $\mathbf{b}$ & SE & t-value & p-value \\
\hline Intercept & -2.230 & 1.495 & -1.492 & .136 \\
Hand & $\mathbf{- 4 . 2 6 1}$ & $\mathbf{1 . 2 7 6}$ & $\mathbf{- 3 . 3 3 9}$ & $\mathbf{. 0 0 1}$ \\
Hand:Position & $\mathbf{4 . 0 7 1}$ & $\mathbf{1 . 5 6 3}$ & $\mathbf{2 . 6 0 4}$ & $\mathbf{. 0 0 9}$
\end{tabular}

680-760 ms, Model 1.2. The same model and approach were used as before but with averaged force in the time window 680-760 ms as a dependent variable. The effect of Hand was significant (larger force in the left hand: $\mathrm{b}=-3.331, \mathrm{p}=.017)$, as well as interaction between Hand and Position $(\mathrm{b}=4.291, \mathrm{p}=$ .012). Note that this time window is a part of a larger one $(260-1000 \mathrm{~ms})$, where this interaction was investigated in detail (see below). The main effect of Position was not significant $(b=0.382, p=.655)$. Marginal r-squared was .026, and conditional r-squared was .473. See Table 2 for further details.

Table 2. Model 1.2. Main effect of Position on grip force in the time window 680-760 ms (Experiment $1)$.

\begin{tabular}{lcccc}
\hline Random effects: & Name & Variance & SD & \\
\hline Participants & Intercept & 102.000 & 10.100 & \\
Residual & & 120.100 & 10.960 & \\
\hline Fixed effects: & $\mathbf{b}$ & SE & t-value & p-value \\
\hline Intercept & -1.692 & 1.725 & -0.981 & .327 \\
Hand & $\mathbf{- 3 . 3 3 1}$ & $\mathbf{1 . 3 9 8}$ & $\mathbf{- 2 . 3 8 4}$ & $\mathbf{. 0 1 7}$
\end{tabular}




$\begin{array}{lllll}\text { Position } & 0.382 & 0.856 & 0.446 & .655 \\ \text { Hand:Position } & 4.291 & 1.712 & 2.507 & .012\end{array}$

60-130 ms, Model 1.3. The data were restructured, and the mean-centered force of the opposite hand in the same time window was included as a predictor ${ }^{1}$. As before, continuously coded Position $(-1=$ left, $0=$ center,$+1=$ right) was included as fixed effect, participants were included as random intercepts. Function drop1 was used to identify and successively eliminate non-significant terms. The effect of Position on the left-hand force (after accounting for the contralateral force) was close to significance with higher force when stars were presented on the left side $(b=-0.681, p=.052$; marginal $r$-squared $=$ .073 , conditional r-squared $=.726$; see Table 3 for details; see also Figure 3 ).

Table 3. Model 1.3. Effect of Position on the left grip force (after controlling for the contralateral force) in the time window 60-130 ms (Experiment 1).

\begin{tabular}{lcccc}
\hline Random effects: & Name & Variance & SD & \\
\hline Participants & Intercept & 22.434 & 4.736 & \\
Residual & & 9.435 & 3.072 & \\
\hline Fixed effects: & b & SE & t-value & p-value \\
\hline Intercept & 4.305 & 0.790 & 5.451 & $<.001$ \\
Contralateral hand & $\mathbf{0 . 2 8 8}$ & $\mathbf{0 . 0 7 4}$ & $\mathbf{3 . 8 9 2}$ & $<.001$ \\
(right) & & & & \\
Position & $\mathbf{- 0 . 6 8 1}$ & $\mathbf{0 . 3 5 0}$ & $\mathbf{- 1 . 9 4 7}$ & $\mathbf{. 0 5 2}$
\end{tabular}

Model 1.4. A similar analysis was run with the right-hand force as a dependent variable and Position and mean-centered left force as fixed predictors. This time a strong effect of Position was found with larger right force when stimuli were presented on the right side $(b=1.316, p=.005$; marginal $r$ squared $=.371$, conditional $\mathrm{r}$-squared $=.408 ;$ see Table 4 for details; see also Figure 3 ).

Table 4. Model 1.4. Effect of Position on the right grip force (after controlling for the contralateral force) in the time window 60-130 ms (Experiment 1).

\begin{tabular}{llll}
\hline Random effects: & Name & Variance & SD \\
\hline
\end{tabular}

\footnotetext{
${ }^{1}$ The force of the opposite hand was used as a predictor, in order to account for the correlation of forces due to automatic coordination between hands (Mathew et al., 2020). I suggest this approach for analysis of lateralized effects (such as in this study) on grip force.
} 


\begin{tabular}{lcccc}
$\begin{array}{l}\text { Participants } \\
\text { Residual }\end{array}$ & Intercept & 1.083 & 1.041 & \\
\hline Fixed effects: & b & 17.588 & 4.194 & \\
\hline Intercept & 3.443 & 0.412 & 8.364 & p-value \\
$\begin{array}{l}\text { Contralateral hand } \\
\text { (left) }\end{array}$ & $\mathbf{0 . 4 9 3}$ & $\mathbf{0 . 0 6 3}$ & $\mathbf{7 . 8 1 1}$ & $<.001$ \\
Position & & & & $\mathbf{0 0 0 1}$ \\
& $\mathbf{1 . 3 1 6}$ & $\mathbf{0 . 4 6 4}$ & $\mathbf{2 . 8 3 9}$ & $\mathbf{. 0 0 5}$
\end{tabular}
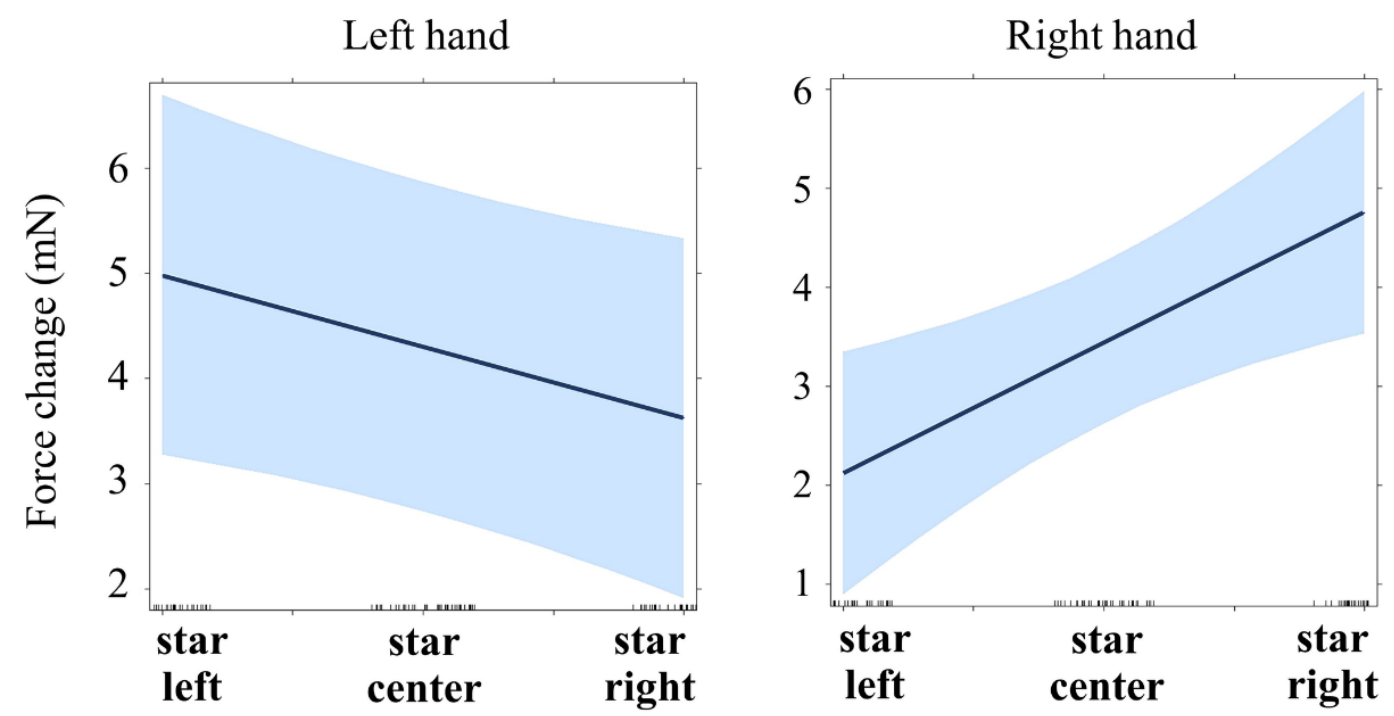

Fig. 3. Regression lines for the main effect of star position on force (Experiment 1, time window 60-130 ms). See main text for details (Models 1.3 and 1.4).

260-1000 ms, Models 1.5 and 1.6. The same approach was used as for the previous time window (60$130 \mathrm{~ms}$ ). Each force was tested separately, with the contralateral force and Position as predictors. This time the effect of Position was significant in both hands: the left force increased when stimuli were presented on the left $(b=-1.989, p=.011 ;$ marginal $r$-squared $=.053$, conditional $r$-squared $=.665$; see Table $\mathrm{N})$ and the right force increased when the stimuli were presented on the right $(\mathrm{b}=2.949, \mathrm{p}<$ .001 ; marginal $r$-squared $=.092$, conditional $r$-squared $=.621$; see Tables 5 and 6 ; see also Figure 4 ).

Table 5. Model 1.5. Effect of Position on the left grip force (after controlling for the contralateral force) in the time window 260-1000 ms (Experiment 1). 


\begin{tabular}{lcccc}
\hline Random effects: & Name & Variance & SD & \\
\hline Participants & Intercept & 82.810 & 9.100 & \\
Residual & & 45.420 & 6.740 & \\
\hline Fixed effects: & $\mathbf{b}$ & SE & t-value & p-value \\
\hline Intercept & 1.420 & 1.546 & 0.919 & .358 \\
$\begin{array}{l}\text { Contralateral hand } \\
\text { (right) }\end{array}$ & $\mathbf{0 . 2 1 7}$ & $\mathbf{0 . 0 8 9}$ & $\mathbf{2 . 4 3 6}$ & $\mathbf{. 0 1 5}$ \\
Position & & & & \\
& $\mathbf{- 1 . 9 8 9}$ & $\mathbf{0 . 7 8 1}$ & $\mathbf{- 2 . 5 4 9}$ & $\mathbf{. 0 1 1}$
\end{tabular}

Table 6. Model 1.6. Effect of Position on the right grip force (after controlling for the contralateral force) in the time window 260-1000 ms (Experiment 1).

\begin{tabular}{lcccc}
\hline Random effects: & Name & Variance & SD & \\
\hline Participants & Intercept & 66.100 & 8.130 & \\
Residual & & 47.340 & 6.881 & \\
\hline Fixed effects: & $\mathbf{b}$ & SE & t-value & p-value \\
\hline Intercept & -1.992 & 1.413 & -1.409 & .159 \\
Contralateral hand & $\mathbf{0 . 2 2 0}$ & $\mathbf{0 . 0 8 6}$ & $\mathbf{2 . 5 4 5}$ & $\mathbf{. 0 1 1}$ \\
(left) & & & & \\
Position & $\mathbf{2 . 9 4 9}$ & $\mathbf{0 . 7 7 0}$ & $\mathbf{3 . 8 3 2}$ & $<.001$
\end{tabular}
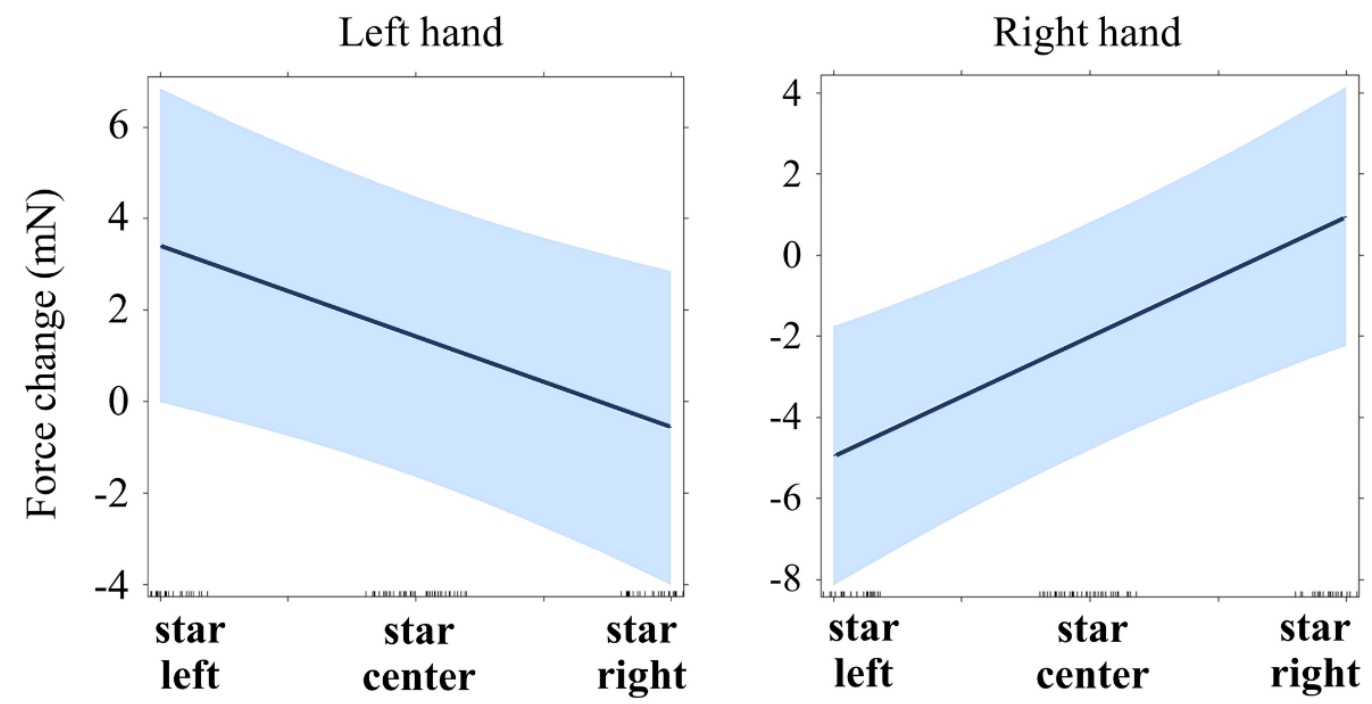

Fig. 4. Regression lines for the main effect of star position on force (Experiment 1, time window

260-1000 ms). See main text for details (Models 1.5 and 1.6). 
To summarize, Experiment 1 demonstrated an early (already at 60-130 ms after stimulus onset) interaction between Hand and Position. However, the effect of lateralized stimuli on grip force was rather asymmetric: the effect of Position was more pronounced in the right hand than the left hand.

\section{Experiment 2: Arrow presentation}

This experiment induced endogenous attentional shifts by centrally presenting arrows (Friesen et al., 2004; Marotta et al., 2018) pointing to the left, right, or both directions, while participants' grip force was recorded bimanually.

\section{Participants}

The same sample of participants as in Experiment 1 participated in this experiment.

\section{Stimuli and design}

Red and yellow arrows were used as stimuli in catch (go) and critical (no-go) trials accordingly. The background was kept black. Stimuli can be found in the supplementary materials (see data availability statement). Arrows always appeared in the middle of the screen and pointed to the left, right, or in both directions. Grip force was recorded bimanually. This results in a 2 (Hand: left / right) X 3 (Direction of the arrow: left / both / right) within-participant design.

\section{Task and procedure}

After the calibration procedure already described above, the experiment started. Each trial consisted of a fixation dot (200 ms), followed by a stimulus (until response, but no longer than $2000 \mathrm{~ms}$ ).

Participants saw arrows around $2 \mathrm{~cm}$ in diameter (1.91 degrees of visual angle) with equal probability $(33 \%)$ pointing into one of three directions (left, right, or both). In $25 \%$ of all trials, red arrows appeared, the other $75 \%$ of arrows were yellow. The task was to say "yes" when a red arrow appeared. Participants were asked not to cross their legs during the experiment. Critical trials were always no-go trials (yellow arrows). The experiment lasted around 15 minutes and consisted of 360 trials with a break in the middle. A short practice (12 trials) preceded the experiment. 


\section{Data preprocessing and analysis}

The same preprocessing procedures were applied as in Experiment 1. One participant had the proportion of trials with force exceeding pre-defined thresholds $( \pm 500 \mathrm{mN})$ larger than $20 \%$ and thus was excluded. For the remaining participants, this proportion ranged from $0 \%$ to $10 \%$ (mean $=2 \%$ ). Those trials were discarded. Among preserved participants, accuracy varied from $99 \%$ to 100\% (mean $=100 \%$ ); error trials were excluded from further analysis.

Force patterns are shown in Figure 5. The solid blue line in Figure 5A represents averaged force of both hands for all accepted no-go trials. Independently of a particular condition, grip force follows a pattern closely resembling that from Experiment 1: there are three peaks (H130, H300, and H650) with two dips between them (L230 and L500). This time, the first peak (H130) is the tallest. Figure 5B represents averaged forces in go (dotted red line) and no-go (solid blue line) trials. As in the first Experiment, these two lines start diverging at $250 \mathrm{~ms}$ after stimulus onset with force in go trials reaching its highest point (around $55 \mathrm{mN}$ ) at around $700 \mathrm{~ms}$ and remaining at this level till the end of the epoch (1000 ms). Figure 5C represents force averaged by condition (Hand X Direction). 


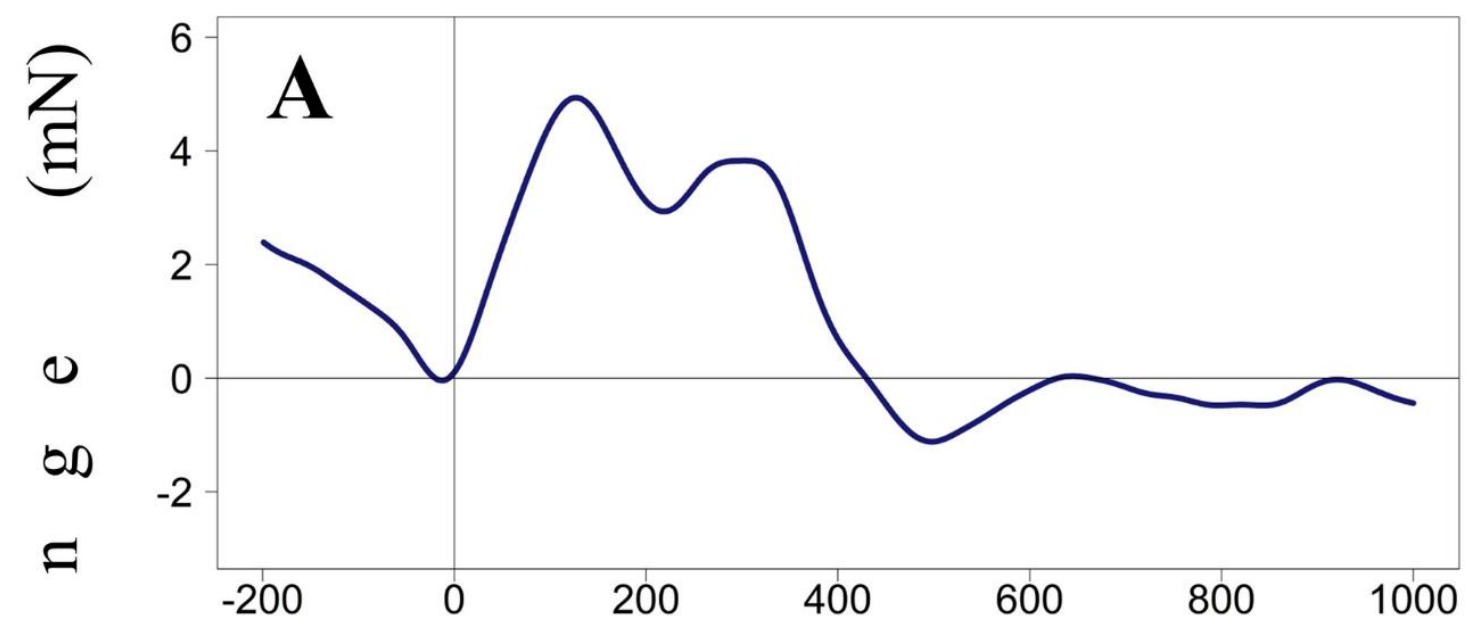

$\sigma$
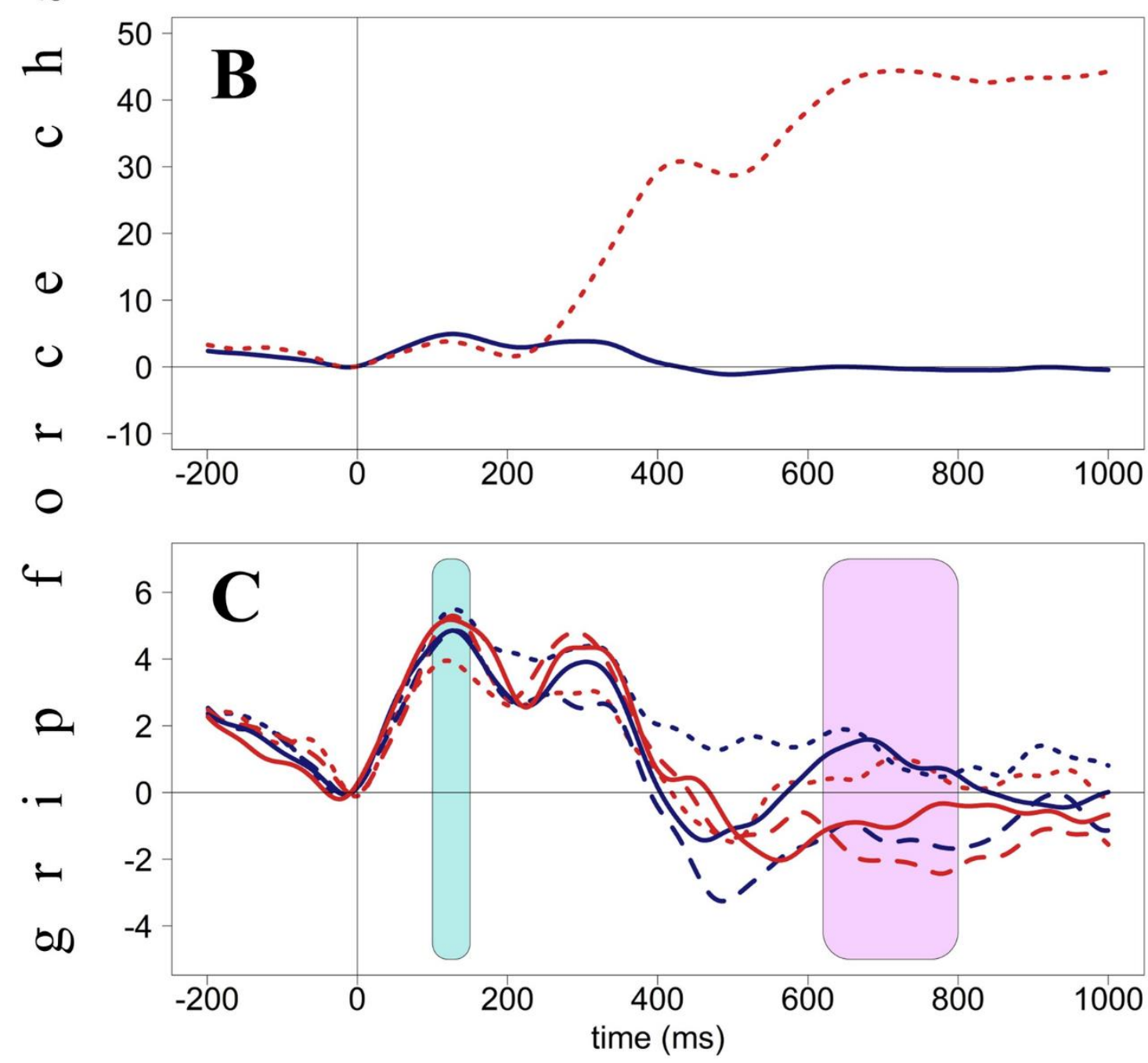

Fig. 5. Grip force changes (in milli-Newton) plotted against time from stimulus onset (in milliseconds), Experiment 2. Panel A. Averaged force profiles across all accepted no-go trials of all participants. Panel B. Force profiles in go (dotted red line) and no-go (solid blue line) trials. These 
forces diverge around $250 \mathrm{~ms}$ after stimulus presentation. Panel C. Force profiles averaged by condition (Hand X Direction). Blue area (100-150 ms) indicates interaction between Hand and Direction; pink area $(620-800 \mathrm{~ms})$ represents the main effect of Direction close to significance $(\mathrm{p}<.1)$. Red lines represent right-hand forces, blue lines - left-hand forces. Dotted lines represent the arrowleft condition, solid lines - arrow-in-both-directions, dashed lines - arrow-right.

As before, I performed a cluster permutation analysis with Hand and Direction as within-variables. Five thousand permutations were performed, and TFCE (Threshold-Free Cluster Enhancement) correction for multiple comparisons was used. The analysis revealed a main effect of Direction close to significance in two time windows (510-580 ms and 620-800 ms) and one time window with an interaction between the variables close to significance (100-150 ms).

510-580 ms, Model 2.1. As in Experiment 1, the data (averaged by Hand and Direction for each participant) were then submitted to linear mixed model analysis. The categorical predictor Hand was sum-coded (left/right, sum-coded contrast -0.5 and 0.5 ). Direction was re-coded in a continuous manner (left: -1; both directions: 0; right: 1). Interaction between Hand and Direction was included. Participants were included as random factors. I performed a backward elimination using the drop1 function to identify the best-fit model; effects and interactions that did not improve model fit $(\mathrm{p}>.1)$ were successively eliminated. Only significant effects are reported unless the effect of interest in a given analysis was non-significant - in such cases, it is also reported.

No significant effects or interactions were revealed in this time window. The closest to significance was the main effect of Direction, as demonstrated before in the cluster permutation analysis. The force (non-significantly) decreased for right-pointing arrows compared to left-pointing arrows $(b=-1.170, p$ $=.100)$. Marginal $r$-squared was .007 , and conditional $r$-squared was .384. See Table 7 for further details. 
Table 7. Model 2.1. Main effect of Direction (not significant) on grip force in the time window 510$580 \mathrm{~ms}$ (Experiment 2).

\begin{tabular}{lcccc}
\hline Random effects: & Name & Variance & SD & \\
\hline Participants & Intercept & 49.62 & 7.044 & \\
Residual & & 80.90 & 8.994 & \\
\hline Fixed effects: & b & SE & t-value & p-value \\
\hline Intercept & -0.772 & 1.256 & -0.615 & 0.539 \\
Direction & $\mathbf{- 1 . 1 7 0}$ & $\mathbf{0 . 7 1 1}$ & $\mathbf{- 1 . 6 4 5}$ & $\mathbf{0 . 1 0 0}$
\end{tabular}

620-800 ms, Model 2.2. The same approach was applied as in the previous time window. The effect of Direction was marginally significant $(\mathrm{p}<.1)$ and thus remained in the model. Again, the force in both hands slightly decreased for right-pointing arrows compared to left-pointing arrows $(b=-1.279, p=$ .089). The effect of Hand and the interaction between the two variables were not significant. Marginal r-squared was .006, and conditional r-squared was .508. See Table 8 for further details.

Table 8. Model 2.2. Main effect of Direction on grip force in the time window 620-800 ms (Experiment 2).

\begin{tabular}{lcccc}
\hline Random effects: & Name & Variance & SD & \\
\hline Participants & Intercept & 92.23 & 9.604 & \\
Residual & & 90.25 & 9.500 & \\
\hline Fixed effects: & b & SE & t-value & p-value \\
\hline Intercept & -0.211 & 1.638 & -0.129 & .898 \\
Direction & $\mathbf{- 1 . 2 7 9}$ & $\mathbf{0 . 7 5 1}$ & $\mathbf{- 1 . 7 0 2}$ & $\mathbf{. 0 8 9}$
\end{tabular}

100-150 ms, Models 2.3 and 2.4. The same approach was used as for Experiment 1 (see time windows 60-130 ms and 260-1000 ms). Direction was re-coded as a continuous variable (left: -1 ; both directions: 0; right: 1). Each force was tested separately, with the contralateral force and Direction as predictors. The effect of Direction was significant in both hands: the left force increased when stimuli arrows pointed to the left $(\mathrm{b}=-0.717, \mathrm{p}=.028$; marginal $\mathrm{r}$-squared $=.599$, conditional $\mathrm{r}$-squared $=$ .810 ; see Table $\mathrm{N})$ and the right force increased when the arrows pointed to the right $(\mathrm{b}=0.867, \mathrm{p}=$ .013 ; marginal r-squared $=.566$, conditional $r$-squared $=.817$; see Tables 9 and 10; see also Figure 6) 
bioRxiv preprint doi: https://doi org/101101/2021.12,28,474381; this version posted December 28,2021 . The copyright holder for this preprint (which was not certified by peer review) is the author/funder, who has granted bioRxiv a license to display the preprint in perpetuity. It is made available under aCC-BY 4.0 International license.

Table 9. Model 2.3. Effect of Direction on the left grip force (after controlling for the contralateral force) in the time window 100-150 ms (Experiment 2).

\begin{tabular}{lcccc}
\hline Random effects: & Name & Variance & SD & \\
\hline Participants & Intercept & 9.364 & 3.060 & \\
Residual & & 8.412 & 2.900 & \\
\hline Fixed effects: & $\mathbf{b}$ & $\mathbf{S E}$ & t-value & p-value \\
\hline Intercept & 4.900 & 0.552 & 8.884 & $<.001$ \\
$\begin{array}{l}\text { Contralateral hand } \\
\text { (right) }\end{array}$ & $\mathbf{0 . 6 4 4}$ & $\mathbf{0 . 0 5 8}$ & $\mathbf{1 1 . 0 8 0}$ & $<.001$ \\
Direction & & & & $\mathbf{0 2 8}$
\end{tabular}

Table 10. Model 2.4. Effect of Direction on the right grip force (after controlling for the contralateral force) in the time window 100-150 ms (Experiment 2).

\begin{tabular}{lcccc}
\hline Random effects: & Name & Variance & SD & \\
\hline Participants & Intercept & 13.303 & 3.647 & \\
Residual & & 9.688 & 3.112 & \\
\hline Fixed effects: & $\mathbf{b}$ & $\mathbf{S E}$ & t-value & p-value \\
\hline Intercept & 4.663 & 0.643 & 7.253 & $<.001$ \\
Contralateral hand & $\mathbf{0 . 7 6 7}$ & $\mathbf{0 . 0 7 2}$ & $\mathbf{1 0 . 6 3 8}$ & $<.001$ \\
(left) & & & & \\
Direction & $\mathbf{0 . 8 6 7}$ & $\mathbf{0 . 3 4 9}$ & $\mathbf{2 . 4 8 6}$ & $\mathbf{. 0 1 3}$
\end{tabular}
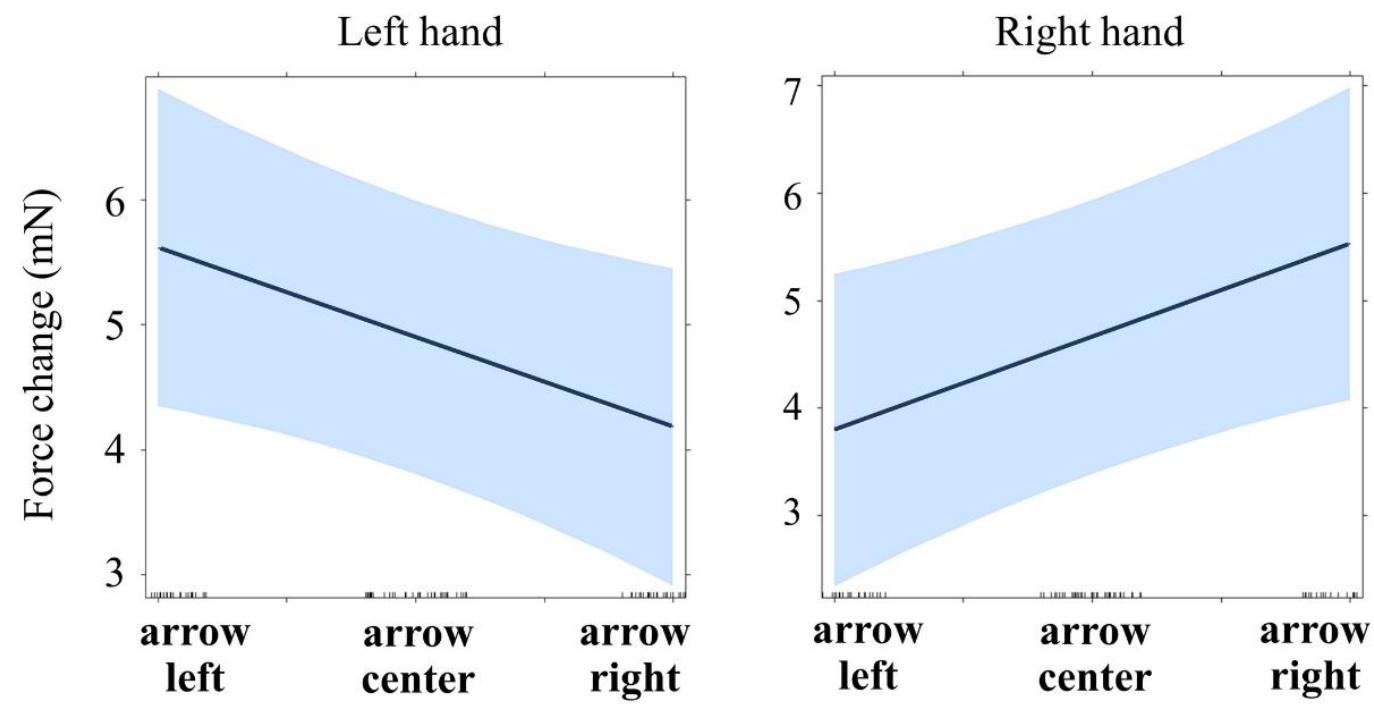


\section{Fig. 6. Regression lines for the main effect of arrow direction on force (Experiment 2, time}

window 100-150 ms). See main text for details (Models 2.3 and 2.4).

Thus, Experiment 2 demonstrated a pattern similar to Experiment 1: left- and right-pointing arrows led to a significant force decrease in the contralateral hand. Unlike in Experiment 1, where the effect was stronger in the right hand, the magnitude of the effect in Experiment 2 was comparable across hands. Moreover, the effect of arrow direction was less pronounced and appeared later than the effect of star position.

\section{Experiment 3: Word presentation}

Words produce attentional effects different than those resulting from arrows. While some authors assumed that both arrows and linguistic cues cause similar endogenous attentional shifts (e.g., Posner, 1980), more recent studies demonstrated essential differences between these types of cues (Ristic \& Kingstone, 2012). A series of studies showed that words with a typical localization in vertical space (e.g., shoe, grass, sun, or cloud) could shift attention in the corresponding direction (Dudschig et al., 2012, 2013; Estes et al., 2008). This effect extends even to abstract concepts, such as god or devil (Chasteen et al., 2010). Simple linguistic cues (e.g., the word up) can also modulate trajectories of vertical saccades (Janyan et al., 2015).

In Experiment 3, participants saw centrally presented words LINKS, RECHTS or ZENTRUM ("left", "right" or "center" in German; Note, however, that words "LINKS" and "RECHTS" are adverbs in German, while the word "ZENTRUM" is a noun). Bimanual force recording allowed to investigate dynamic involvement of the motor system into the processing of spatial information presented in a purely symbolic way, i.e., through linguistic meaning.

\section{Participants}


Only a subsample of German native speakers participated in this experiment $(\mathrm{N}=27$; mean age $=24$;

9 males and 18 females). On average, participants spoke 1.85 foreign languages, most frequently

English, Spanish, and French. The mean EHI score of those participants was +60 , with 21 participants (78\%) having EHI score $>+50,2$ participants (7\%) having EHI scores between +50 and -50 , and 4 participants $(15 \%)$ with EHI score < -50. All participants reported normal or corrected-to-normal vision. No participant took medications affecting motor control.

\section{Stimuli and design}

Red and yellow words or meaningless symbol arrays (e.g., §@\#\$\%) were used as stimuli in catch (go) and critical (no-go) trials accordingly. The background was kept black. Experimental scripts can be found in the supplementary data (see data availability statement). Grip force was recorded bimanually. This results in a 2 (Hand: left / right) X 3 (Word: left / center / right) within-participant design.

\section{Task and procedure}

After the calibration procedure described above, the experiment started. Each trial consisted of a fixation dot (200 ms), followed by a stimulus (until response, but no longer than $2000 \mathrm{~ms}$ ). Participants saw words or stimuli arrays having a length of around $2.5 \mathrm{~cm}$ (2.39 degrees of visual angle). See Table 1 for exact proportions of stimuli of each type. Words "left", "right", and "center" appeared with equal probability. The task was to say "yes" when a red word (20\% of all trials) or symbol array (20\% of all trials) appeared. Participants were asked not to cross their legs during the experiment. Critical trials were always no-go with words (i.e., only real words in yellow font, $40 \%$ of all trials). In the remaining $20 \%$ of all trials, yellow symbol arrays appeared, and no response was required. The experiment consisted of 450 trials with a break in the middle and lasted around 20 minutes. A short practice (18 trials) preceded the experiment.

\section{Data preprocessing and analysis}


The same preprocessing procedures were applied as in Experiment 1. The proportion of trials with force exceeding pre-defined thresholds $( \pm 500 \mathrm{mN})$ ranged from $0 \%$ to $18 \%($ mean $=2 \%)$, and no participant was excluded due to this criterion. Those trials were discarded. Accuracy varied from 97\% to $100 \%$ (mean $=99 \%)$; error trials were excluded from further analysis.

Figure 7A represents averaged force of both hands for all accepted no-go trials plotted for words only. Independently of condition, grip force demonstrates the following pattern: there are two well-defined peaks of equal height (H130 and H350) with a dip between them (L200) and a slight deviation of force at the beginning of the second peak (H250). After H350, the force drops dramatically until $600 \mathrm{~ms}$ and remains relatively stable until the end of the epoch $(1000 \mathrm{~ms})$, with only a tiny wave having its peak at H850. Figure 7B represents averaged forces in go (dotted red line) and no-go (solid blue line) trials. These two lines start diverging at $230 \mathrm{~ms}$ after stimulus onset with force in go trials reaching its highest point (around $40 \mathrm{mN}$ ) at around $650 \mathrm{~ms}$ and remaining at this level till the end of the epoch. Figure 7C represents force averaged by condition (Hand X Word). 

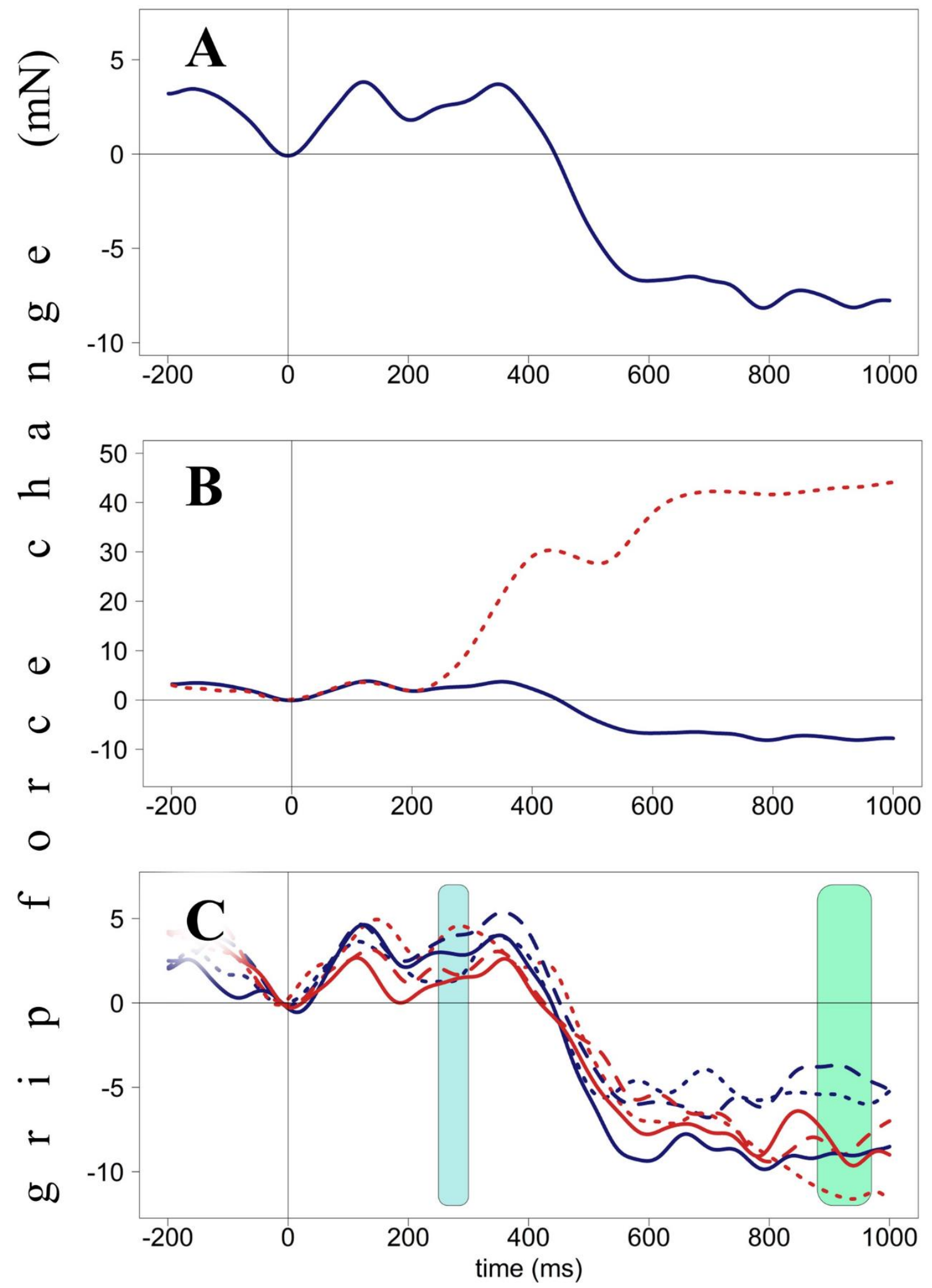

Fig. 7. Grip force changes (in milli-Newton) plotted against time from stimulus onset (in

milliseconds), Experiment 3. Panel A. Averaged force profiles across all accepted no-go trials of all participants plotted for words. Panel B. Force profiles in go (dotted red line) and no-go (solid blue 
line) trials. These forces diverge around $230 \mathrm{~ms}$ after stimulus presentation. Panel C. Force profiles averaged by condition (Hand X Word). Blue area (830-860 ms) indicates interaction between Hand and Word. Green area (880-970 ms) indicates the main effect of Hand (not significant). Red lines represent right-hand forces, blue lines - left-hand forces. Dotted lines represent the word-left condition, solid lines - word-center, dashed lines - word-right.

As before, a cluster permutation analysis with Hand and Word as within-variables and their interaction was used to identify time windows of interest. Five thousand permutations were performed, and TFCE (Threshold-Free Cluster Enhancement) correction for multiple comparisons was used. The analysis revealed a main effect of Hand close to significance (880-970 ms) and two time windows with an interaction between Hand and Word close to significance (250-300 ms and 810-890 ms).

880-970 ms, Model 3.1. As in Experiments 1 and 2, the data (averaged by Hand and Word for each participant) were then submitted to linear mixed model analysis. The categorical predictor Hand was sum-coded (left/right, sum-coded contrast -0.5 and 0.5 ). Word was re-coded in a continuous manner (left: -1; center: 0; right: 1). Interaction between Hand and Word was included. Participants were included as random factors. I performed a backward elimination using the drop1 function to identify the best-fit model; effects and interactions that did not improve model fit ( $p>.1)$ were successively eliminated. Only significant effects are reported unless the effect of interest in a given analysis was non-significant - in such cases, it is also reported.

No significant effects or interactions were revealed in this time window. The closest to significance was the main effect of Hand. The force was (non-significantly) lower in the left hand compared to the right hand $(b=-3.355, p=.097)$. Marginal $r$-squared was .013, and conditional $r$-squared was .249. See Table 11 for further details.

Table 11. Model 3.1. Main effect of Hand on grip force in the time window 880-970 ms (not significant; Experiment 3). 


\begin{tabular}{lcccc}
\hline Random effects: & Name & Variance & SD & \\
\hline Participants & Intercept & 52.2 & 7.225 & \\
Residual & & 165.9 & 12.882 & \\
\hline Fixed effects: & $\mathbf{b}$ & SE & t-value & p-value \\
\hline Intercept & -7.866 & 1.720 & -4.574 & .001 \\
Hand & $\mathbf{- 3 . 3 5 5}$ & $\mathbf{2 . 0 2 4}$ & $\mathbf{- 1 . 6 5 7}$ & $\mathbf{. 0 9 7}$
\end{tabular}

250-300 ms, Models 3.2 and 3.3. The same approach was used as for Experiment 1 (see time windows 60-130 ms and 260-1000 ms). Word was re-coded as a continuous variable (left: -1; center: 0; right: 1). Each force was tested separately, with the contralateral force and Word as predictors. The effect of Word was significant in the left hand: the grip force increased for the word "right" compared to the word "left" $(\mathrm{b}=1.736, \mathrm{p}=.047$; marginal $\mathrm{r}$-squared $=.171$, conditional $\mathrm{r}$-squared $=.764$; see Table 12), and in the right hand the force increased for the word "left" compared to the word "right" ( $b=$ $1.690, \mathrm{p}=.041$; marginal $\mathrm{r}$-squared $=.166$, conditional $\mathrm{r}$-squared $=.768$; see Table 13 ; see also Figure 8).

Table 12. Model 3.2. Effect of Direction on the left grip force (after controlling for the contralateral force) in the time window 250-300 ms (Experiment 3).

\begin{tabular}{lcccc}
\hline Random effects: & Name & Variance & SD & \\
\hline Participants & Intercept & 101.23 & 10.061 & \\
Residual & & 40.39 & 6.355 & \\
\hline Fixed effects: & $\mathbf{b}$ & $\mathbf{S E}$ & t-value & p-value \\
\hline Intercept & 2.761 & 2.061 & 1.339 & .180 \\
Contralateral hand & $\mathbf{0 . 3 9 7}$ & $\mathbf{0 . 1 0 8}$ & $\mathbf{3 . 6 8 0}$ & $<.001$ \\
(right) & & & & $\mathbf{1 . 9 8 3}$ \\
Word & $\mathbf{1 . 7 3 6}$ & $\mathbf{0 . 8 7 5}$ & $\mathbf{0 4 7}$
\end{tabular}

Table 13. Model 3.3. Effect of Direction on the right grip force (after controlling for the contralateral force) in the time window 250-300 ms (Experiment 3).

\begin{tabular}{lcccc}
\hline Random effects: & Name & Variance & SD & \\
\hline Participants & Intercept & 94.34 & 9.713 & \\
Residual & & 36.25 & 6.021 & \\
\hline Fixed effects: & b & SE & t-value & p-value \\
\hline Intercept & 2.502 & 1.985 & 1.260 & .208 \\
Contralateral hand & $\mathbf{0 . 3 5 8}$ & $\mathbf{0 . 0 9 8}$ & $\mathbf{3 . 6 4 8}$ & $<.001$ \\
(left) & & & &
\end{tabular}




\section{$\begin{array}{lllll}\text { Word } & -1.690 & 0.828 & -2.041 & .041\end{array}$}
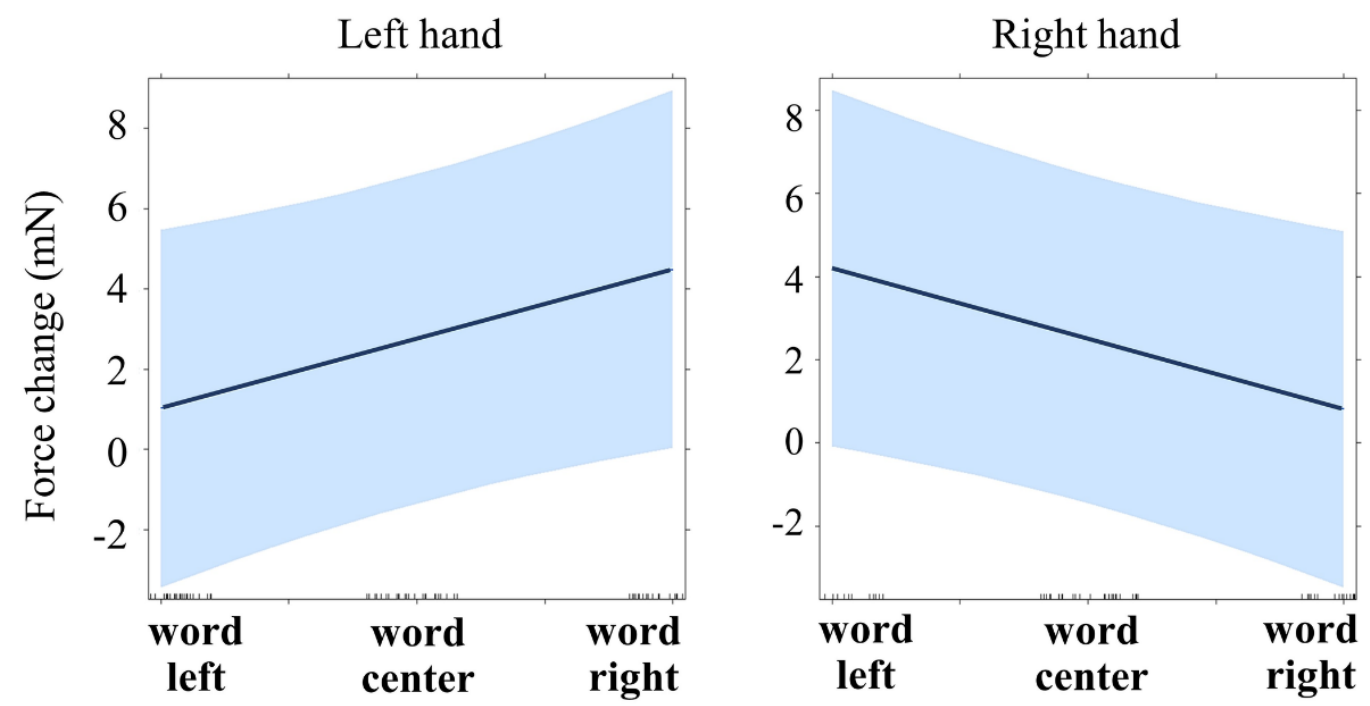

Fig. 8. Regression lines for the main effect of word semantics on force (Experiment 3, time window 250-300 ms). See main text for details (Models 3.2 and 3.3).

810-890 ms, Models 3.4 and 3.5. The same approach was used as for the previous time window. The effect of Word was not significant in the left hand $(b=0.121, p=.938$; marginal $r$-squared $=.210$, conditional $\mathrm{r}$-squared $=.501$; see Table 14$)$, neither was it significant in the right hand $(\mathrm{b}=0.638, \mathrm{p}=$ $.619 ;$ marginal $\mathrm{r}$-squared $=.188$, conditional $\mathrm{r}$-squared $=.596$; see Table 15 ).

Table 14. Model 3.4. Effect of Direction on the left grip force (after controlling for the contralateral force) in the time window 810-890 ms (Experiment 3).

\begin{tabular}{lcccc}
\hline Random effects: & Name & Variance & SD & \\
\hline Participants & Intercept & 75.94 & 8.715 & \\
Residual & & 130.15 & 11.408 & \\
\hline Fixed effects: & $\mathbf{b}$ & $\mathbf{S E}$ & t-value & p-value \\
\hline Intercept & -6.270 & 2.102 & -2.982 & .003 \\
$\begin{array}{l}\text { Contralateral hand } \\
\text { (right) }\end{array}$ & $\mathbf{0 . 5 0 6}$ & $\mathbf{0 . 1 1 2}$ & $\mathbf{4 . 5 2 3}$ & $<.001$ \\
Word & & & & $\mathbf{0 . 0 7 8}$ \\
\end{tabular}


Table 15. Model 3.5. Effect of Direction on the right grip force (after controlling for the contralateral force) in the time window 810-890 ms (Experiment 3).

\begin{tabular}{lcccc}
\hline Random effects: & Name & Variance & SD & \\
\hline Participants & Intercept & 89.61 & 9.466 & \\
Residual & & 88.60 & 9.413 & \\
\hline Fixed effects: & $\mathbf{b}$ & $\mathbf{S E}$ & t-value & p-value \\
\hline Intercept & -8.538 & 2.101 & -4.064 & $<.001$ \\
Contralateral hand & $\mathbf{0 . 4 0 8}$ & $\mathbf{0 . 0 8 6}$ & $\mathbf{4 . 7 6 2}$ & $<.001$ \\
$\begin{array}{l}\text { (left) } \\
\text { Word }\end{array}$ & $\mathbf{0 . 6 3 8}$ & $\mathbf{1 . 2 8 2}$ & $\mathbf{0 . 4 9 8}$ & $\mathbf{. 6 1 9}$
\end{tabular}

Thus, the effect of Word emerged at 250-300 ms, which is later than the effect of Position (Experiment 1) or Direction (Experiment 2). Moreover, the grip force increased in hand contralateral to the expected one: in the right hand for the word "left" and in the left hand for the word "right". This surprising finding will be discussed in detail in the last section.

\section{Analysis at the individual level across experiments}

Since the same individuals participated in all three experiments, it was possible to analyze data across studies. Note that this analysis is rather exploratory due to the small number of participants. I added random slopes to all models where significant interaction between Hand and Side (Position, Direction, or Word) emerged, for left and right hands separately: 60-130 ms and 260-1000 ms (Experiment 1), 100-150 ms (Experiment 2), and 250-300 ms (Experiment 3). These individual coefficients were submitted into a correlational analysis (see Table 16).

Table 16. Correlations between individual slopes across three experiments. Numbers in variable names denote time windows. $\mathrm{S}$ - stars (Experiment 1); $\mathrm{A}$ - arrows (Experiment 2); $\mathrm{W}$ - words (Experiment 3). LH - left-hand force; $\mathrm{RH}$ - right-hand force. $\mathrm{N}$ - number of participants included in the analysis (varied across experiments). $* \mathrm{p}<.05 ; * * \mathrm{p}<.01 ; * * * \mathrm{p}<.001$.

\begin{tabular}{|c|c|c|c|c|c|c|c|}
\hline & $\begin{array}{l}\text { S 60-130 } \\
\text { RH }\end{array}$ & $\begin{array}{l}\text { S 260- } \\
1000 \mathrm{LH}\end{array}$ & $\begin{array}{l}\text { S 260- } \\
1000 \mathrm{RH}\end{array}$ & $\begin{array}{l}\text { A 100- } \\
150 \mathrm{LH}\end{array}$ & $\begin{array}{l}\text { A 100- } \\
150 \mathrm{RH}\end{array}$ & $\begin{array}{l}\text { W 250- } \\
300 \text { LH }\end{array}$ & $\begin{array}{l}\text { W 250- } \\
300 \mathrm{RH}\end{array}$ \\
\hline $\mathrm{N}$ & 41 & 41 & 41 & 40 & 40 & 26 & 26 \\
\hline
\end{tabular}




\begin{tabular}{|c|c|c|c|c|c|c|c|}
\hline S 60-130 LH & $\begin{array}{r}-.268 \\
\mathrm{p}=.090\end{array}$ & $\begin{array}{r}-.013 \\
\mathrm{p}=.936\end{array}$ & $\begin{array}{r}-.030 \\
\mathrm{p}=.853\end{array}$ & $\begin{array}{r}-.300 \\
p=.061\end{array}$ & $\begin{array}{c}-.495 * * \\
p=.001\end{array}$ & $\mathrm{p}=.250$ & $\mathrm{p}=.394$ \\
\hline S 60-130 RH & & $\begin{array}{r}-.394 * \\
\mathbf{p}=.011\end{array}$ & $\begin{array}{l}.625 * * * \\
\mathbf{p}<.001\end{array}$ & $\mathrm{p}=\begin{array}{r}.070 \\
.670\end{array}$ & $\begin{array}{r}.479 * * \\
\mathrm{p}=.002\end{array}$ & $\mathrm{p}=.257$ & $\begin{array}{c}-.435 * \\
\mathrm{p}=.026\end{array}$ \\
\hline S 260-1000 LH & & & $\begin{array}{c}-.438 * * \\
p=.004\end{array}$ & $p=\begin{array}{r}.147 \\
.366\end{array}$ & $\begin{array}{r}-.286 \\
\mathrm{p}=.074\end{array}$ & $\begin{array}{l}-.510 * * \\
\mathbf{p}=.008\end{array}$ & $\mathrm{p}=.944$ \\
\hline S 260-1000 RH & & & & $\mathrm{p}=.253$ & $\mathrm{p}=\begin{array}{r}.174 \\
.283\end{array}$ & $p=.259$ & $\begin{array}{r}-.278 \\
p=.170\end{array}$ \\
\hline A $100-150 \mathrm{LH}$ & & & & & $\begin{array}{r}-.402 * \\
p=.010\end{array}$ & $\begin{array}{r}-.289 \\
p=.152\end{array}$ & $\begin{array}{r}-.111 \\
\mathrm{p}=.591\end{array}$ \\
\hline A $100-150 \mathrm{RH}$ & & & & & & $=.336$ & $\begin{array}{r}-.177 \\
\mathrm{p}=.388\end{array}$ \\
\hline W 250-300 LH & & & & & & & $\begin{array}{l}.21 \\
.28\end{array}$ \\
\hline
\end{tabular}

Correlations between hands in the same time windows within the same experiments. A negative significant correlation $(\mathrm{r}=-.438, \mathrm{p}=.004)$ was found between the right-hand and left-hand coefficients in the time window 260-1000 ms (Experiment 1, star presentation). Since the effect of Side is oppositely directed in the left and right hands, this negative correlation demonstrates that both forces were influenced by the stimuli simultaneously at the individual level. In other words, the same participants, who demonstrated the effect in one hand, were also more likely to demonstrate it in another hand. The same pattern was found for the time window 100-150 ms in Experiment 2 (arrow presentation; $\mathrm{r}=-.402, \mathrm{p}=.010$ ). The correlation for the time window $60-130 \mathrm{~ms}$ in Experiment 1 (star presentation) was close to significance $(\mathrm{r}=-.268, \mathrm{p}=.090)$. The correlation for the time window 250-300 $\mathrm{ms}$ in Experiment 3 (word presentation) was also negative, though far from significance $(\mathrm{r}=$ $.217, \mathrm{p}=.288)$. Overall, these results show that Position and Direction have simultaneous effects on both hands at the individual level. For Word, this cannot be concluded, perhaps due to absence of the effect or a smaller number of participants in Experiment $3(\mathrm{~N}=26)$.

Correlations across time windows within the same experiment. More interesting is the positive correlation between right-hand slope coefficients for the time window 60-130 ms and 260-1000 ms (Experiment 1, star presentation; $\mathrm{r}=.625, \mathrm{p}<.001$ ). This correlation suggests that the effect of 
Position observed in the earlier time window $(60-130 \mathrm{~ms})$ and in the later one $(260-1000 \mathrm{~ms})$ is substantially the same effect that was interrupted between 130-260 ms, probably due to large force oscillations (H130 and L230, see Experiment 1). No such correlation was observed for the left-hand coefficients $(\mathrm{r}=-.013, \mathrm{p}=.936)$.

Correlations across experiments. Right-hand coefficients in the time window 60-130 ms, Experiment 1, correlated positively with right-hand coefficients in the time window 100-150 ms, Experiment $2(\mathrm{r}=$ $.479, \mathrm{p}=.002)$. A negative correlation was observed for left-hand coefficients in the time window 60 $130 \mathrm{~ms}$, Experiment 1, and right-hand coefficients in the time window 100-150 ms, Experiment $2(\mathrm{r}=$ $-.495, \mathrm{p}=.001)$. These correlations demonstrate that the same participants, who showed the effect of Position in Experiment 1 (star presentation), were more likely to exhibit a comparable effect of Direction in Experiment 2 (arrow presentation). More surprising are the other two negative correlations: the one between right-hand coefficients at 60-130 ms in Experiment 1 and at 250-300 ms in Experiment $3(\mathrm{r}=-.435, \mathrm{p}=.026)$; and the other one between left-hand coefficients at 260-1000 $\mathrm{ms}$ in Experiment 1 and at 250-300 ms at Experiment $3(-.510, \mathrm{p}=.008)$. These two correlations indicate that the same participants who demonstrated the effect of Position in Experiment 1 (star presentation) also demonstrated the effect of Word in Experiment 3, although the effect of Word was the opposite of the expected.

In the next section, all results will be discussed in more detail.

\section{DISCUSSION}

The present study aimed to investigate the effects of spatial processing on the manual motor system by using a new method - bimanual grip force recording. In Experiment 1, participants' visual attention was shifted using lateralized stimuli presentation (Experiment 1). In Experiment 2, participants were centrally presented with pictographic symbols with spatial meaning (left- or right-oriented arrows). In Experiment 3, participants were centrally presented with words having spatial meaning ("left" vs. 
"right"). Since a go/no-go paradigm with a verbal response in go trials was used, any observed effects can only be attributed to spatial/semantic processing alone and not to motor preparation of responses.

General pattern of grip force changes. The first important finding is that all three types of stimuli (stars, arrows, and words) led to very similar initial force patterns (see Figure 9), namely two peaks (around 130 and 300-350 ms after stimulus onset) followed by differentially declining force profiles. I will now interpret these results in turn.

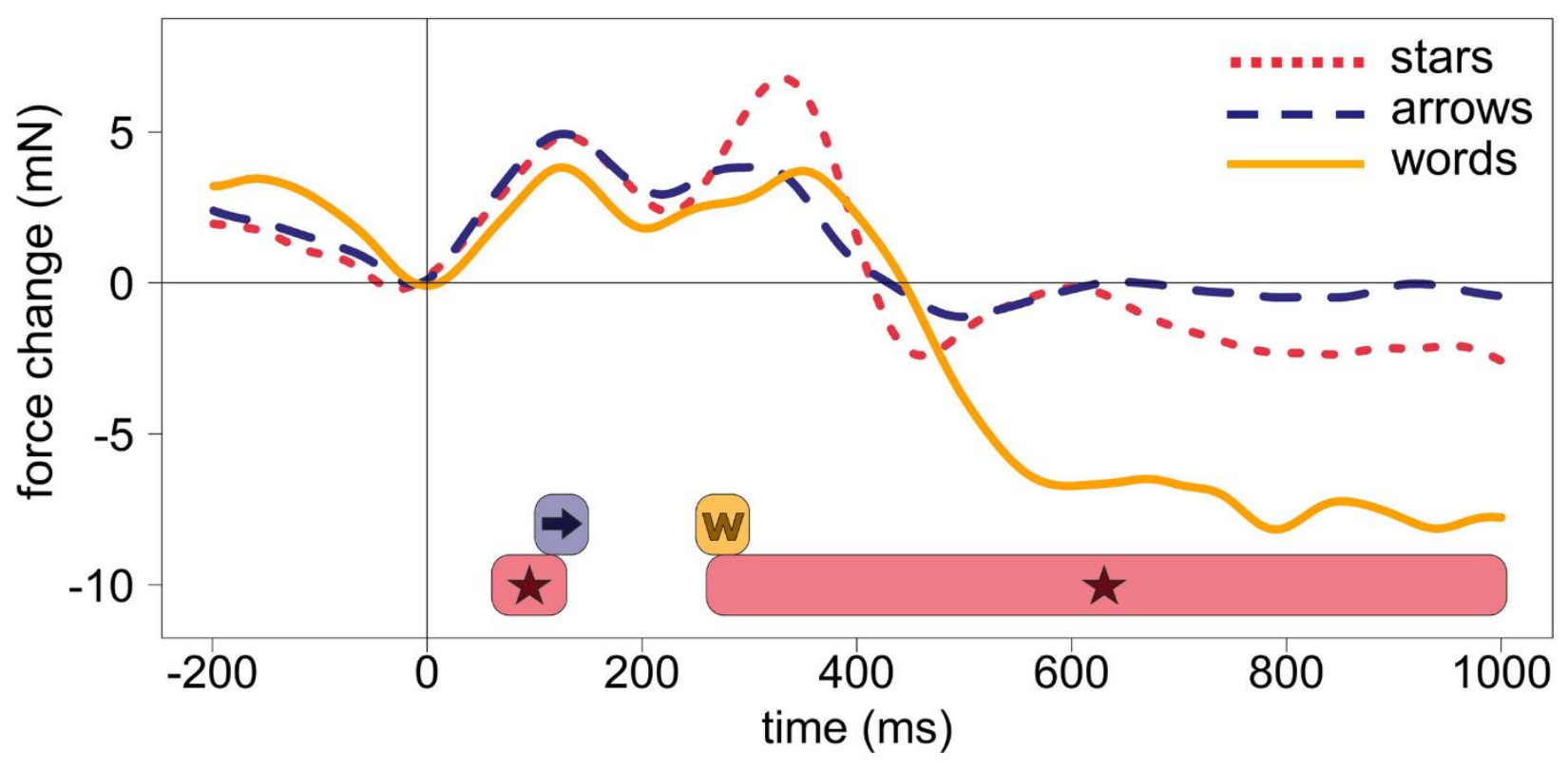

Figure 9. Averaged forces from both hands across all no-go trials (regardless of condition) in three experiments. The dotted red line represents force modulations caused by the presentation of stars (Experiment 1), dashed blue line - arrows (Experiment 2), solid yellow line - words (Experiment 3). Colored horizontal bars in the lower part of the graph represent time windows with significant Side X Hand interactions for all three types of stimuli - stars, arrows, and words. The colors of bars correspond to the colors of lines described above.

This pattern is very close to force profiles observed in studies with other stimuli - numbers (Miklashevsky et al., 2021) and human faces (Miklashevsky et al., in prep.). In response to numbers, 
grip force peaked at $100 \mathrm{~ms}$ and later at $350 \mathrm{~ms}$. Two more prominent peaks (around 100-130 and 300$350 \mathrm{~ms}$ ) probably reflect two processing stages: (1) initial processing of any stimulus appearing on a screen and (2) decision-making or response inhibition process accordingly. Remember that only no-go trials are included in analyses. The divergence point between forces (230-250 ms) in go and no-go trials always precedes the second peak - i.e., at this point, participants' arousal increases in trials requiring response and should be inhibited in trials where no response is needed. These assumptions require confirmation in future studies.

Interestingly, a slight deviation formed an additional peak at $250 \mathrm{~ms}$ in an earlier study with numbers (Miklashevsky et al., 2021) which results in a pattern very similar to those observed for words and (less pronounced) arrows in the present study but not for stars (in this study) or faces (in a previous study, unpublished). Since numbers, words, and arrows are all symbolic stimuli, I hypothesize that this smaller peak around $250 \mathrm{~ms}$ reflects some process specific to the understanding of symbols.

Suppose my hypothesis is correct and grip force reflects not only motor-related but also more general cognitive processes, such as stimulus identification and preparation or inhibition of verbal responses. In that case, this has implications for other studies using the force registration method. In previous studies of this kind (e.g., Frak et al., 2010; Nazir et al., 2017), force changes were always interpreted as specific signatures of activity in the manual motor system. However, a recent study shows that even observing foot actions leads to changes in grip force (Blampain et al., 2018), perhaps due to automatic propagation of activity in the motor brain areas. The same principle might be at play in the present study. Thus, it might be methodologically incorrect to choose force at the time point of stimulus onset as a baseline and compare different conditions with it since any stimulus might lead to force oscillations. Different conditions should be compared with each other instead, or a continuous coding of variables should be chosen as in the present study.

Influence of spatial processing on grip force changes. Regarding the specific hypothesis of the present study, i.e., whether or not spatial attentional shifts lead to the automatic activation of the 
manual motor system - the answer is yes, although with some reservations. When lateralized stimuli appeared (stars, Experiment 1), a significant difference in force emerged already at 60-130 ms and later at 260-1000 ms. The force increased in each of the hands when the star was presented on the ipsilateral side and decreased for stars presented on the contralateral side. This effect was stronger in the right hand in both time windows. Since individual linear coefficients for both time windows (60$130 \mathrm{~ms}$ and $260-1000 \mathrm{~ms}$ ) correlated positively for the right hand, I suppose these two time windows reflect the same effect. The interruption between the two windows appears due to the multiphasic structure of force profiles: forces in all conditions go down rapidly between 130 and $230 \mathrm{~ms}$, thus reducing differences between conditions. Note that no significant effects were found in all three experiments in this time interval.

A similar pattern, although less pronounced, appeared for arrows (Experiment 2): the force increased in each of the hands for arrows pointing in that hand's direction and decreased for arrows pointing in the opposite direction. This effect emerged later than (at $100 \mathrm{~ms}$ ), lasted shorter (just $50 \mathrm{~ms}$ ), and was weaker than for stars. Nevertheless, it was also found in the first "wave" (H130), i.e., it probably belongs to the same processing stage. It is not surprising since symbolic cues have a similar impact on attention as physical ones, even if these symbolic cues are task-irrelevant (see Hommel et al., 2001; Ranzini et al., 2009). The present study provides even more substantial evidence in favor of the automatic effect of arrows on the attentional system than the studies by Hommel, Pratt et al. (2001), or Ranzini et al. (2009). In these previous studies, the arrows themselves were non-predictive, but lateralized stimuli still followed arrows. In the present study, no lateralized stimuli were used (Experiment 2), yet force patterns resembled those produced by exogenous attentional shifts. The effect for arrows (Experiment 2), measured as regression coefficients, correlated with the effect for stars (Experiment 1) at the individual level, indicating stable inter-individual differences in motor response to spatial stimuli.

Arrows are viewed as symbolic stimuli in the present study, although there is a debate about their actual status: arrows demonstrate effects typical for both exogenous and endogenous cues. Ristic and 
Kingstone even speak of the third form of attention - automated symbolic orienting (Ristic \&

Kingstone, 2012). In the present study, the general force pattern for arrows is similar to both: the pattern produced by words (the second peak, H300, is lower than the first one, H130), but also to the pattern produced by stars (grip force does not drop as deeply as for words after 300-350 ms and there is a slight increase around $600-650 \mathrm{~ms}$ ). The same is true regarding the effect of spatial information: qualitatively similar to those produced by stars, it is at the same time weaker for arrows. It appears later than for stars but earlier than for words.

A significant effect of word semantics on force was found in Experiment 3. Yet, the direction of this effect was surprising: the force increased for words related to the opposite side and decreased for words related to the same side. The effect appeared rather late (250-300 ms) and was of comparable strength across both hands. Note that it belongs to the second larger force wave and emerges simultaneously with the tiny force deviation discussed above (H250). This information might be crucial for interpreting the direction of the effect: remember that participants inhibited their verbal response in no-go trials, and the divergence point between go and no-go trials, presumably reflecting the moment of such inhibition, was exactly $230 \mathrm{~ms}$. The stronger H250 wave probably results from the conflict between the two overlapping processes: automatic activation of word semantics and conscious inhibition of verbal response. By suppressing semantics of the word "left", participants literally had to inhibit activity in the left hand; the same happened with the word "right" and the right hand. If this is the case, this finding has implications for the research on inhibitory control: multiple measures of this presumably higher-order construct often do not correlate with each other (e.g., Gärtner \& Strobel, 2021). The reason might be that inhibitory control does not constitute a single construct but is instead highly domain-specific.

The findings of the present study are in line with neuroscientific research of attention. ERP research established a fine-graded time course of attentional processes. Attentional effects on the processing of lateralized stimuli were found in the magnitude of P1 (60-100 ms after stimulus onset), N1 (around $150 \mathrm{~ms}$ ), and P2 (around $200 \mathrm{~ms}$ ) ERP components (see for review Luck et al., 2000). Effects of 
symbolic control of attention induced by using centrally presented arrows appear at 200-400 ms after cue onset over contralateral posterior sites (the so-called early directing attention negativity, EDAN), probably reflecting encoding spatial information provided by the cue. EDAN follows by anterior directing attention negativity (ADAN) appearing over contralateral anterior sites at 300-500 ms and performing attentional shift. Finally, a positive waveform (late directing attention positivity, LDAP) appears after $500 \mathrm{~ms}$ after cue onset and presumably represents top-down modulation in the excitability of sensory areas ( see for review Eimer et al., 2002; also Ranzini et al., 2009). This observation aligns with reaction time research demonstrating the highest performance in detecting targets around $300 \mathrm{~ms}$ after the onset of a symbolic cue. Still, this effect appears even earlier (see Klein, 2020, for discussion). The present study confirms very early effects of both lateralized visual stimuli (60-130 ms) and centrally presented symbolic cues (100-150 ms). It also demonstrates that these effects are detectable in the manual motor system. Such early and automatic modulation is one of the signatures of functional coupling between two structures and not mere spreading spillover activation (see Pulvermüller, 2012).

Although the present study shares many similarities with classical research using the Posner paradigm, substantial differences in the setup and procedure should be considered when comparing the current results with previous studies. First, no laterally appearing stars, arrows, or words were cues in the original sense of this term: there were no "target" stimuli following them. Instead, they were themselves the targets. While lateralized stars indeed led to automatic attentional shifts due to their location, symbolic cues (arrows and words) could indirectly shift attention, following automatic processing of their meaning, which was not necessary for the color discrimination task. The task itself required merely superficial processing (Craik \& Lockhart, 1972) and was not related to spatial information. These factors taken together make the similarity between effects of lateralized stimuli and centrally presented arrows even more remarkable. Further research should vary the role of stimuli (by turning them into cues with varying validity as in the original Posner paradigm) and complexity of the 
motor task to clarify the exact functional relationship between the attentional and manual motor systems (cf. Ibáñez \& García, 2018).

\section{Conclusion}

The present study investigated the relationship between spatial attention and the manual motor system by using a go/no-go paradigm and bimanual registration of grip force. Automatic and rapid changes in grip force were found in response to lateralized visual stimuli (Experiment 1) and centrally presented symbolic stimuli (Experiments 2, arrows, and 3, words). This activity followed a similar early biphasic pattern for all kinds of stimuli: one peak emerged at $130 \mathrm{~ms}$ and another at 300-350 ms after stimulus onset. For both lateralized objects and centrally presented arrows, the direction of the effect was as predicted, i.e., the left force increased in response to objects presented on the left side or left-pointing arrows, while the opposite was true for the right force. This effect appeared very early for lateralized objects $(60 \mathrm{~ms})$ and slightly later for arrows $(100 \mathrm{~ms})$. A reverse pattern was observed for words: each of the two forces increased for words related to the opposite side. The effect for words was significant at 250-300 ms after stimulus onset. This surprising finding might indicate an interaction between automatic semantic activation and inhibition of verbal responses required in no-go trials. Overall, the results suggest a close relationship between attentional processes and the manual motor system. Further research should clarify the functional role of the manual motor system activation in processing spatial information.

\section{Acknowledgments}

I thank Martin H. Fischer for his valuable feedback on the early versions of this manuscript.

\section{Data Availability Statement}

The datasets generated for this study can be found in the Open Science Framework (OSF) at https://doi.org/10.17605/OSF.IO/H6P7M.

\section{References}


Abrams, R. A., Davoli, C. C., Du, F., Knapp, W. H., \& Paull, D. (2008). Altered vision near the hands. Cognition, 107(3), 1035-1047. https://doi.org/10.1016/j.cognition.2007.09.006

Adam, J. J., Bovend'Eerdt, T. J. H., van Dooren, F. E. P., Fischer, M. H., \& Pratt, J. (2012). The closer the better: Hand proximity dynamically affects letter recognition accuracy. Attention, Perception, \& Psychophysics, 74(7), 1533-1538. https://doi.org/10.3758/s13414-012-0339-3

Aravena, P., Courson, M., Frak, V., Cheylus, A., Paulignan, Y., Deprez, V., \& Nazir, T. (2014). Action relevance in linguistic context drives word-induced motor activity. Frontiers in Human Neuroscience, 8. https://doi.org/10.3389/fnhum.2014.00163

Aravena, P., Delevoye-Turrell, Y., Deprez, V., Cheylus, A., Paulignan, Y., Frak, V., \& Nazir, T. (2012). Grip Force Reveals the Context Sensitivity of Language-Induced Motor Activity during “Action Words" Processing: Evidence from Sentential Negation. PLOS ONE, 7(12), e50287. https://doi.org/10.1371/journal.pone.0050287

Baldauf, D., \& Deubel, H. (2008). Visual attention during the preparation of bimanual movements. Vision Research, 48(4), 549-563. https://doi.org/10.1016/j.visres.2007.11.023

Baldauf, D., Wolf, M., \& Deubel, H. (2006). Deployment of visual attention before sequences of goaldirected hand movements. Vision Research, 46(26), 4355-4374. https://doi.org/10.1016/j.visres.2006.08.021

Barr, D. J., Levy, R., Scheepers, C., \& Tily, H. J. (2013). Random effects structure for confirmatory hypothesis testing: Keep it maximal. Journal of Memory and Language, 68(3), 255-278. https://doi.org/10.1016/j.jml.2012.11.001

Bates, D., Mächler, M., Bolker, B., \& Walker, S. (2015). Fitting Linear Mixed-Effects Models Using Ime4. Journal of Statistical Software, 67(1). https://doi.org/10.18637/jss.v067.i01

Blampain, J., Ott, L., \& Delevoye-Turrell, Y. N. (2018). Seeing action simulation as it unfolds: The implicit effects of action scenes on muscle contraction evidenced through the use of a gripforce sensor. Neuropsychologia, 114, 231-242. https://doi.org/10.1016/j.neuropsychologia.2018.04.026 
Brockmole, J. R., Davoli, C. C., Abrams, R. A., \& Witt, J. K. (2013). The World Within Reach: Effects of Hand Posture and Tool Use on Visual Cognition. Current Directions in Psychological Science, 22(1), 38-44. https://doi.org/10.1177/0963721412465065

Chapman, C. S., Gallivan, J. P., Wood, D. K., Milne, J. L., Culham, J. C., \& Goodale, M. A. (2010). Reaching for the unknown: Multiple target encoding and real-time decision-making in a rapid reach task. Cognition, 116(2), 168-176. https://doi.org/10.1016/j.cognition.2010.04.008

Chasteen, A. L., Burdzy, D. C., \& Pratt, J. (2010). Thinking of God moves attention. Neuropsychologia, 48(2), 627-630. https://doi.org/10.1016/j.neuropsychologia.2009.09.029

Craik, F. I. M., \& Lockhart, R. S. (1972). Levels of processing: A framework for memory research. Journal of Verbal Learning and Verbal Behavior, 11(6), 671-684. https://doi.org/10.1016/S0022-5371(72)80001-X

da Silva, R. L., Labrecque, D., Caromano, F. A., Higgins, J., \& Frak, V. (2018). Manual action verbs modulate the grip force of each hand in unimanual or symmetrical bimanual tasks. PLoS ONE, 13(2). https://doi.org/10.1371/journal.pone.0192320

da Silva, R. L., Santos, F. F., Mendes, I. M. G., Caromano, F. A., Higgins, J., \& Frak, V. (2019). Contributions of the Left and the Right Hemispheres on Language-Induced Grip Force Modulation of the Left Hand in Unimanual Tasks. Medicina, 55(10), 674. https://doi.org/10.3390/medicina55100674

Davoli, C. C., \& Brockmole, J. R. (2012). The hands shield attention from visual interference. Attention, Perception, \& Psychophysics, 74(7), 1386-1390. https://doi.org/10.3758/s13414012-0351-7

Davoli, C. C., Du, F., Montana, J., Garverick, S., \& Abrams, R. A. (2010). When meaning matters, look but don't touch: The effects of posture on reading. Memory \& Cognition, 38(5), 555-562. https://doi.org/10.3758/MC.38.5.555 
Davoli, C. C., O’Rear, C. D., McAulay, E., McNeil, N. M., \& Brockmole, J. R. (2020). Hand position affects performance on multiplication tasks. Journal of Numerical Cognition, 6(1), 1-21. https://doi.org/10.5964/jnc.v6i1.211

Dehaene, S., Bossini, S., \& Giraux, P. (1993). The mental representation of parity and number magnitude. Journal of Experimental Psychology: General, 122(3), 371-396. https://doi.org/10.1037/0096-3445.122.3.371

Dudschig, C., Lachmair, M., de la Vega, I., De Filippis, M., \& Kaup, B. (2012). From top to bottom: Spatial shifts of attention caused by linguistic stimuli. Cognitive Processing, 13 Suppl 1, S151154. https://doi.org/10.1007/s10339-012-0480-x

Dudschig, C., Souman, J., Lachmair, M., Vega, I. de la, \& Kaup, B. (2013). Reading “Sun” and Looking Up: The Influence of Language on Saccadic Eye Movements in the Vertical Dimension. PLoS ONE, 8(2), e56872. https://doi.org/10.1371/journal.pone.0056872

Eimer, M., Velzen, J. van, \& Driver, J. (2002). Cross-Modal Interactions between Audition, Touch, and Vision in Endogenous Spatial Attention: ERP Evidence on Preparatory States and Sensory Modulations. Journal of Cognitive Neuroscience, 14(2), 254-271. https://doi.org/10.1162/089892902317236885

Estes, Z., Verges, M., \& Barsalou, L. W. (2008). Head up, foot down: Object words orient attention to the objects' typical location. Psychological Science, 19(2), 93-97. https://doi.org/10.1111/j.1467-9280.2008.02051.x

Festman, Y., Adam, J. J., Pratt, J., \& Fischer, M. H. (2013). Continuous hand movement induces a farhand bias in attentional priority. Attention, Perception, \& Psychophysics, 75(4), 644-649. https://doi.org/10.3758/s13414-013-0430-4

Fischer, M. H. (2003). Spatial representations in number processing-evidence from a pointing task. Visual Cognition, 10(4), 493-508. https://doi.org/10.1080/13506280244000186 
Fischer, M. H., \& Shaki, S. (2014). Spatial Associations in Numerical Cognition-From Single Digits to Arithmetic. Quarterly Journal of Experimental Psychology, 67(8), 1461-1483. https://doi.org/10.1080/17470218.2014.927515

Frak, V., Nazir, T., Goyette, M., Cohen, H., \& Jeannerod, M. (2010). Grip force is part of the semantic representation of manual action verbs. PloS One, 5(3), e9728. https://doi.org/10.1371/journal.pone.0009728

Friesen, C. K., Ristic, J., \& Kingstone, A. (2004). Attentional Effects of Counterpredictive Gaze and Arrow Cues. Journal of Experimental Psychology: Human Perception and Performance, 30(2), 319-329. https://doi.org/10.1037/0096-1523.30.2.319

Frossard, J., \& Renaud, O. (2018). Permutation tests for regression, ANOVA and comparison of signals: The permuco package. 27.

Gallivan, J. P., \& Chapman, C. S. (2014). Three-dimensional reach trajectories as a probe of real-time decision-making between multiple competing targets. Frontiers in Neuroscience, 8. https://doi.org/10.3389/fnins.2014.00215

Gärtner, A., \& Strobel, A. (2021). Individual Differences in Inhibitory Control: A latent Variable Analysis. Journal of Cognition, 4(1), 17. https://doi.org/10.5334/joc.150

Hommel, B., Chapman, C. S., Cisek, P., Neyedli, H. F., Song, J.-H., \& Welsh, T. N. (2019). No one knows what attention is. Attention, Perception, \& Psychophysics, 81(7), 2288-2303. https://doi.org/10.3758/s13414-019-01846-w

Hommel, B., Pratt, J., Colzato, L., \& Godijn, R. (2001). Symbolic Control of Visual Attention. Psychological Science, 12(5), 360-365. https://doi.org/10.1111/1467-9280.00367

Ibáñez, A., \& García, A. M. (2018). Context as Inter-domain Effects: The Hand-Action-Network Dynamic Language Embodiment Model. In A. Ibáñez \& A. M. García (Eds.), Contextual Cognition: The Sensus Communis of a Situated Mind (pp. 29-54). Springer International Publishing. https://doi.org/10.1007/978-3-319-77285-1_3 
Janyan, A., Vankov, I., Tsaregorodtseva, O., \& Miklashevsky, A. (2015). Remember down, look down, read up: Does a word modulate eye trajectory away from remembered location? Cognitive Processing, 16(S1), 259-263. https://doi.org/10.1007/s10339-015-0718-5

Klein, R. M. (2004). On the Control of Visual Orienting. In Cognitive neuroscience of attention (pp. 29-44). The Guilford Press.

Klein, R. M. (2020). Is covert spatial orienting embodied or disembodied cognition? A historical review. Quarterly Journal of Experimental Psychology, 73(1), 20-28. https://doi.org/10.1177/1747021819889497

Krause, F., \& Lindemann, O. (2014). Expyriment: A Python library for cognitive and neuroscientific experiments. Behavior Research Methods, 46(2), 416-428. https://doi.org/10.3758/s13428013-0390-6

Luck, S. J., Woodman, G. F., \& Vogel, E. K. (2000). Event-related potential studies of attention. Trends in Cognitive Sciences, 4(11), 432-440. https://doi.org/10.1016/S1364-6613(00)01545$\mathrm{X}$

Marotta, A., Román-Caballero, R., \& Lupiáñez, J. (2018). Arrows don’t look at you: Qualitatively different attentional mechanisms triggered by gaze and arrows. Psychonomic Bulletin \& Review, 25(6), 2254-2259. https://doi.org/10.3758/s13423-018-1457-2

Mathew, J., de Rugy, A., \& Danion, F. R. (2020). How optimal is bimanual tracking? The key role of hand coordination in space. Journal of Neurophysiology, 123(2), 511-521. https://doi.org/10.1152/jn.00119.2019

Mathôt, S., Schreij, D., \& Theeuwes, J. (2012). OpenSesame: An open-source, graphical experiment builder for the social sciences. Behavior Research Methods, 44(2), 314-324. https://doi.org/10.3758/s13428-011-0168-7

Miklashevsky, A., Lindemann, O., \& Fischer, M. H. (2021). The Force of Numbers: Investigating Manual Signatures of Embodied Number Processing. Frontiers in Human Neuroscience, 14, 590508. https://doi.org/10.3389/fnhum.2020.590508 
Myachykov, A., Ellis, R., Cangelosi, A., \& Fischer, M. H. (2016). Ocular drift along the mental number line. Psychological Research, 80(3), 379-388. https://doi.org/10.1007/s00426-015$0731-4$

Nakagawa, S., \& Schielzeth, H. (2013). A general and simple method for obtaining $R^{2}$ from generalized linear mixed-effects models. Methods in Ecology and Evolution, 4(2), 133-142. https://doi.org/10.1111/j.2041-210x.2012.00261.x

Nazir, T. A., Hrycyk, L., Moreau, Q., Frak, V., Cheylus, A., Ott, L., Lindemann, O., Fischer, M. H., Paulignan, Y., \& Delevoye-Turrell, Y. (2017). A simple technique to study embodied language processes: The grip force sensor. Behavior Research Methods, 49(1), 61-73. https://doi.org/10.3758/s13428-015-0696-7

Oldfield, R. C. (1971). The assessment and analysis of handedness: The Edinburgh inventory. Neuropsychologia, 9(1), 97-113. https://doi.org/10.1016/0028-3932(71)90067-4

Pérez-Gay Juárez, F., Labrecque, D., \& Frak, V. (2019). Assessing language-induced motor activity through Event Related Potentials and the Grip Force Sensor, an exploratory study. Brain and Cognition, 135, 103572. https://doi.org/10.1016/j.bandc.2019.05.010

Posner, M. I. (1980). Orienting of attention. Quarterly Journal of Experimental Psychology, 32(1), 325. https://doi.org/10.1080/00335558008248231

Pulvermüller, F. (2012). Meaning and the brain: The neurosemantics of referential, interactive, and combinatorial knowledge. Journal of Neurolinguistics, 25(5), 423-459.

https://doi.org/10.1016/j.jneuroling.2011.03.004

R Core Team. (2020). R: A language and environment for statistical computing. In $R$ Foundation for Statistical Computing. https://www.R-project.org/

Ranzini, M., Dehaene, S., Piazza, M., \& Hubbard, E. M. (2009). Neural mechanisms of attentional shifts due to irrelevant spatial and numerical cues. Neuropsychologia, 47(12), 2615-2624. https://doi.org/10.1016/j.neuropsychologia.2009.05.011 
Reed, C. L., Grubb, J. D., \& Steele, C. (2006). Hands up: Attentional prioritization of space near the hand. Journal of Experimental Psychology: Human Perception and Performance, 32(1), 166177. https://doi.org/10.1037/0096-1523.32.1.166

Ristic, J., \& Kingstone, A. (2012). A new form of human spatial attention: Automated symbolic orienting. Visual Cognition, 20(3), 244-264. https://doi.org/10.1080/13506285.2012.658101

Rizzo, J.-R., Hosseini, M., Wong, E. A., Mackey, W. E., Fung, J. K., Ahdoot, E., Rucker, J. C., Raghavan, P., Landy, M. S., \& Hudson, T. E. (2017). The Intersection between Ocular and Manual Motor Control: Eye-Hand Coordination in Acquired Brain Injury. Frontiers in Neurology, 8, 227. https://doi.org/10.3389/fneur.2017.00227

Robinson, J. (2013). Edinburgh Handedness Inventory. In F. R. Volkmar (Ed.), Encyclopedia of Autism Spectrum Disorders (pp. 1051-1054). Springer. https://doi.org/10.1007/978-1-44191698-3_877

Schwarz, W., \& Müller, D. (2006). Spatial Associations in Number-Related Tasks. Experimental Psychology, 53(1), 4-15. https://doi.org/10.1027/1618-3169.53.1.4

Shaki, S., \& Fischer, M. H. (2018). Deconstructing spatial-numerical associations. Cognition, 175, 109-113. https://doi.org/10.1016/j.cognition.2018.02.022

Toomarian, E. Y., \& Hubbard, E. M. (2018). On the genesis of spatial-numerical associations: Evolutionary and cultural factors co-construct the mental number line. Neuroscience \& Biobehavioral Reviews, 90, 184-199. https://doi.org/10.1016/j.neubiorev.2018.04.010

Wood, G., Willmes, K., Nuerk, H.-C., \& Fischer, M. H. (2008). On the cognitive link between space and number: A meta-analysis of the SNARC effect. Psychology Science, 50(4), 489-525. 